\title{
La démarche d'investigation dans le cadre des nouveaux programmes de sciences physiques et chimiques : étude de cas au collège
}

The investigation approach as part of the new chemistry and physics syllabus:a case study in collège (lower secondary education)

El trabajo de investigación en el marco de los nuevos programas de ciencias físicas y químicas : estudio de caso en el colegio Der Untersuchungsprozess im Rahmen der neuen Lehrpläne für Chemie und Physik: eine Fallstudie am Collège

\section{Patrice Venturini et Andrée Tiberghien}

\section{(2) OpenEdition}

Journals

Édition électronique

URL : http://journals.openedition.org/rfp/3810

DOI : $10.4000 /$ rfp.3810

ISSN : 2105-2913

Éditeur

ENS Éditions

\section{Édition imprimée}

Date de publication : 15 juillet 2012

Pagination : $95-120$

ISBN : 978-2-84788-388-6

ISSN : 0556-7807

Référence électronique

Patrice Venturini et Andrée Tiberghien, «La démarche d'investigation dans le cadre des nouveaux programmes de sciences physiques et chimiques : étude de cas au collège », Revue française de pédagogie [En ligne], 180 | juillet-août-septembre 2012, mis en ligne le 15 juillet 2015, consulté le 19 avril 2019. URL : http://journals.openedition.org/rfp/3810 ; DOI : 10.4000/rfp.3810 


\title{
La démarche d'investigation dans le cadre des nouveaux programmes de sciences physiques et chimiques: étude de cas au collège
}

\author{
Patrice Venturini et Andrée Tiberghien
}

Cet article concerne la mise en œuvre des nouveaux programmes de sciences physiques au collège. Après leur brève caractérisation, il propose une analyse didactique d'une séance d'enseignement menée par un jeune enseignant en début de carrière. Cette séance est fondée sur une démarche d'investigation, un des éléments les plus importants apparus lors de la rénovation de ces programmes. Cette analyse est réalisée à l'aide de la théorie de l'action conjointe en didactique. Elle fait apparaître à la fois la volonté de cet enseignant de mettre en œuvre cette réforme et ses difficultés à le faire, qu'il surmonte en assumant seul la plus grande partie de l'avancée des savoirs. Une discussion sur les déterminants de l'action didactique (assujettissements institutionnels, épistémologie pratique, rapports personnels) permet de prolonger cette étude de cas par une réflexion plus générale quant à l'application de ces programmes.

Mots-clés (TESE) : secondaire inférieur, action, conduite de la classe, enseignement, physique, réforme des programmes d'étude.

L'étude de cas que nous présentons ici concerne la mise en œuvre des nouveaux programmes d'enseignement de la physique réalisée par un jeune enseignant de collège en début de carrière. Plus particulièrement, nous étudions la mise en œuvre de la démarche d'investigation qu'il propose à ses élèves à propos de la loi d'additivité des tensions dans un circuit série. Cette démarche constitue un des éléments les plus significatifs introduits dans les programmes de sciences du secondaire. Cette méthode pédagogique, supposée notamment intéresser les élèves à cette discipline, les inciter ultérieurement à davantage embrasser une carrière scientifique et leur permettre d'apprendre le fonctionnement des sciences, est apparue d'abord à l'école primaire, puis au collège (MEN, 2007, 2008) et maintenant au lycée.

Nous présenterons d'abord des éléments de cette réforme au niveau du collège ainsi que les questions auxquelles ils nous ont conduits: la démarche d'investigation rompt en effet avec les modalités traditionnelles d'enseignement de la physique et sa mise en œuvre à partir de la prescription des textes officiels interroge. Répondre à ces interrogations nous a amenés à analyser des pratiques effectives d'enseignement. En nous fondant sur des travaux existants sur la démarche d'investigation dont nous proposerons une brève synthèse, nous avons choisi pour nos analyses la théorie de l'action conjointe en didactique (Sensevy, 2007, 2009, 2011) que nous présenterons, associée ensuite à la méthodologie que nous avons utilisée. Toutes deux nous ont permis de caractériser les pratiques de cet enseignant. Nous décrirons ces résultats et les discuterons en proposant des hypothèses tentant de dépasser cette étude de cas, avant de conclure. 


\section{DE NOUVEAUX PROGRAMMES DE COLLĖGE EN SCIENCES PHYSIQUES DONT LA MISE EN CEUVRE INTERROGE...}

Venturini et Tiberghien (2012) ont analysé les programmes de sciences physiques et chimiques du secondaire, et notamment ceux du collège. Selon eux, si dans le cas du collège, les contenus ont très peu varié par rapport aux programmes précédents tout comme, du reste, le temps dédié à leur enseignement, les nouveaux textes mettent fortement en avant l'usage de la démarche d'investigation. L'introduction de cette démarche s'inscrit dans le contexte plus large de l'Inquiry-Based Science Education (IBSE), "inquiry » étant défini par Linn, Davis et Bell $(2004$, p. 4) comme: «the intentional process of diagnosing problems, critiquing experiments, distinguishing alternatives, planning investigations, researching conjectures, searching for information, constructing models, debating with peers and forming coherent arguments. In science inquiry projects, students communicate about scientific topics, evaluate scientific texts, conduct investigations, ask questions about science or technology policies, create designs and critique arguments, often using technology resources. "

Cette définition n'est pas unique et les différents curricula dans le monde mettent plus ou moins l'accent sur l'un ou l'autre de ces aspects. La déclinaison française de cette démarche comporte sept moments décrits dans le texte (MEN, 2007, p. 6):

- le choix d'une situation problème par le professeur; - l'appropriation du problème par les élèves;

- la formulation de conjectures, d'hypothèses explicatives, de protocoles possibles;

- l'investigation ou la résolution du problème conduite par les élèves;

- l'échange argumenté autour des propositions élaborées;

- l'acquisition et la structuration des connaissances;

- l'opérationnalisation des connaissances.

Par ailleurs, les textes font aussi état de connaissances et de compétences que chaque élève est censé maîtriser en fin de collège. C'est dire que la démarche doit bien aboutir in fine à une appropriation de concepts scientifiques. Sans présumer des intentions du législateur, on comprend que cette démarche n'est pas sans lien avec une perspective socioconstructiviste de construction des connaissances, notamment au travers des interactions sociales liées aux activités collectives qui sont évoquées et à la place qu'y occupe le langage. On comprend aussi qu'elle n'est pas sans lien avec une partie de l'activité scientifique développée dans les laboratoires, même si bien sûr, lien ne signifie pas identité ou recouvrement.

Le changement est donc de taille par rapport à un enseignement plus traditionnel de la physique dans lequel l'expérience de monstration (Joshua, 1989, p. 34) avait une place prépondérante. Conçue pour identifier facilement les éléments du réel pertinents pour le savoir en jeu, imposée aux élèves, elle leur permettait sous la conduite étroite de l'enseignant d'induire le modèle à apprendre qui était institutionnalisé. Avec la démarche d'investigation, il s'agit notamment pour le professeur de faire en sorte que les élèves passent d'une situation de départ ouverte et motivante à une question " scientifique " en lien avec le savoir qu'il est censé enseigner (MEN, 2007, p. 7). II s'agit aussi pour lui d'amener les élèves à formuler un modèle explicatif hypothétique, fut-il naïf, permettant de faire des prévisions testées ensuite à travers une expérience qu'ils ont à imaginer. Les élèves doivent enfin être en mesure de présenter et de discuter les résultats obtenus pour préparer les étapes de débat puis d'institutionnalisation et d'opérationnalisation des connaissances que l'enseignant conduira pour terminer. Les deux démarches d'enseignement sont presque aux antipodes l'une de l'autre alors qu'elles partagent les mêmes contraintes de temps et de contenu à enseigner. La réforme n'est donc pas anodine dans la mesure où elle concerne en effet simultanément les trois aspects susceptibles d'être en jeu dans une innovation: les aspects matériels (changement dans les outils, comme les expériences ou les situations), les aspects conceptuels (changement dans la démarche d'enseignement), les aspects relationnels (changements dans les relations interpersonnelles dues aux changements dans les rôles réciproques des enseignants et des élèves) (Cros, 1997, p. 141, d'après Huberman, 19731).

Par ailleurs, Gather Thurler signale les difficultés que rencontrent les réformes liées à la redéfinition des rôles et fonctions des acteurs du système éducatif et/ou effectuées uniquement par le biais de textes ministériels. C'est bien là notre cas tant il est vrai que la formation continue tient une place de plus en plus réduite dans l'accompagnement des évolutions de notre système éducatif. Ce type de réforme ne parviendrait pas à " développer chez les enseignants la volonté d'apprendre et le goût du risque qui sont indispensables pour changer " et le changement visé serait alors " réduit à sa plus simple expression, exécuté de manière grossière ou rejeté dès lors que les premières difficultés paraissent » (Gather Thurler, 2000, p. 29). Mais accepter de prendre des risques même en l'absence de formation ne suffirait pas. Crahay, Wanlin, Issaieva et alii (2010, 
p. 111) concluent en effet, en référence à différentes recherches, que "des changements au niveau des pratiques doivent être accompagnés de changements au niveau des croyances " (ou des connaissances) de l'enseignant, celles-ci agissant comme des filtres à l'égard de nouvelles recommandations institutionnelles et en limitant donc la portée.

Finalement, la nature et l'importance du changement demandé aux enseignants de sciences physiques et chimiques au collège et les conditions dans lesquelles il est opéré interrogent quant à la réalité actuelle de la classe, après quelques années de mise en œuvre. Qu'en est-il exactement? Bien sûr, posée comme telle, la question est bien vaste et notre ambition est seulement d'y apporter quelques éléments de réponse sous forme d'hypothèses générées à partir d'une étude de cas. Celle-ci, qui a une vocation heuristique, implique donc de décrire l'action ordinaire du professeur et de ses élèves dans la classe, associée à l'enseignement d'un savoir particulier à l'aide de la démarche d'investigation. Cette étude s'inscrit donc à la suite de toutes celles qui ont été menées sur l'IBSE.

\section{UNE ÉTUDE INSCRITE DANS UNE LONGUE SÉRIE DE TRAVAUX SUR L'IBSE}

Constituant le point central des réformes dans l'enseignement des sciences depuis les années soixante aux États-Unis (Yager, 1997) et soixante-dix en Europe, I'IBSE a en effet déjà fait l'objet de très nombreux travaux concernant notamment:

- l'analyse des curriculums, standards, programmes pour l'enseignement scientifique pour caractériser les démarches proposées (Dell'Angelo, Coquidé \& Magneron, 2012; Venturini \& Tiberghien, 2012);

- l'identification de références possibles pour les démarches proposées, en lien avec les pratiques sociales de la recherche (Grandy \& Duschl, 2007) ou avec l'apprentissage et l'enseignement des sciences (Mathé, Méheut \& De Hosson, 2008);

- les représentations des enseignants sur la démarche elle-même (Monod-Ansaldi \& Prieur, 2011);

- les croyances et connaissances des enseignants sur la nature des sciences, sur leur enseignement et leur apprentissage (Gess-Newsome, 2002 ; Pélissier \& Venturini, 2012);

- la mise en œuvre de l'IBSE, généralement examinée à partir de l'utilisation de vidéos d'activités en classe.
Dans ce dernier cas, que nous développons car en relation avec notre étude, le focus peut-être mis:

- sur la mise en œuvre elle-même; ce sont alors la nature de l'investigation et son degré d'authenticité qui sont analysés (Chinn \& Malhotra, 2002; Schwartz, Lederman \& Crawford, 2004), les liens qu'entretient l'investigation avec la nature des sciences (Sandoval, 2005) ou encore avec le contexte particulier dans lequel elle est développée, comme celui des questions socio-scientifiques (Walker \& Zeidler, 2007) ou de l'argumentation (Sampson, Grooms \& Walker, 2011);

- sur les élèves; il s'agit alors de comprendre, à partir de leurs difficultés ce qu'ils doivent maîtriser pour mener à bien les activités proposées (Krajcik, Blumenfeld, Marx et al., 1998). Il s'agit encore d'étudier les compétences plus spécifiques qu'ils mettent en jeu, liées par exemple à la mobilisation de concepts particuliers (Reid \& Yang, 2002), à des modalités de représentation (Wu \& Krajcik, 2006) ou encore à la collaboration avec leurs pairs (Watson, Swain \& McRobbie, 2004), éventuellement instrumentée par des logiciels (Makitalo-Siegl, Kohnle \& Fischer, 2011);

- sur l'enseignant; ce sont alors les nouvelles pratiques qui font suite à l'introduction de l'IBSE dans le curriculum qui sont décrites (Blanchard, Southerland, Osborne et al., 2010; Smithenry, 2010), les rôles du professeur qui sont précisés, les interactions au cours des activités d'investigation qui sont caractérisées. Plusieurs analyses concernent des actions particulières comme le questionnement adressé aux élèves ou les réponses qui leur sont apportées, tandis que d'autres touchent simultanément aux actions de l'enseignant et des élèves (Shimoda, White \& Frederiksen, 2002). Des travaux de même type concernent l'analyse du contrat didactique (Van Zee, 2000; Furtak, 2006). Ces études permettent de mettre à jour les contraintes qui pèsent sur la mise en œuvre de l'IBSE et ses limites (Roehrig \& Luft, 2004). De manière plus générale, elles montrent aussi l'intérêt d'étudier de manière simultanée l'action des élèves et de l'enseignant pour mieux comprendre l'une et l'autre.

Cette dernière conclusion nous amène à mobiliser la théorie de l'action conjointe en didactique (TACD) (Sensevy, 2007, 2009, 2011) que nous allons maintenant présenter et qui nous permettra aussi de définir plus précisément notre question de recherche. Notons auparavant que ce bref panorama montre que nos préoccupations sont à la fois partagées sur le plan international et originales puisque liées à un contexte français peu exploré, en particulier en ce qui concerne l'action ordinaire de l'enseignant. 


\section{ANALYSE DE L'ACTION DIDACTIQUE: LA THÉORIE DE L'ACTION CONJOINTE}

La TACD (Sensevy, 2007, 2009) a pour objectif de rendre compte à la fois des dimensions socio-historique et située des pratiques d'enseignement et d'apprentissage en modélisant les transactions entre les différents acteurs survenant à l'occasion de la transmission d'un savoir particulier (Ligozat \& Schubauer-Leoni, 2009, p. 88-91). Elle concerne donc le processus de construction de signification par les élèves à travers ces pratiques.

Sensevy (2009, p. 113, se référant à Wittgenstein, $\left.1997^{2}\right)$ considère d'une manière générale que les actions effectuées en situation selon la logique de la pratique mettent en jeu à la fois la maîtrise de jeux de langage spécifiques, un processus sémiotique particulier et l'émission d'inférences conjointes à partir de l'environnement. Toujours selon lui, une manière de conceptualiser ce processus est de considérer qu'il se déroule "in institutions (Douglas, 19873, 19964) which produce thought collective and thought styles (Fleck, 19345) ». Ce point de vue permet d'envisager la modélisation des pratiques (ici d'enseignement apprentissage) en termes de jeux. Cette perspective est renforcée par le point de vue de Bourdieu (19926) auquel se réfère aussi Sensevy (2009). En effet, la logique de la pratique étant incorporée dans la logique des situations qui la constituent, on peut considérer qu'un individu qui agit dans une partie du monde social (et donc construit à cette occasion du sens à travers la pratique) joue un certain jeu dans des situations liées à des institutions. Le jeu est un modèle qui permet de relier situation et institution. En suivant ce point de vue, les situations liées aux pratiques d'enseignement et d'apprentissage se déroulant dans des institutions particulières peuvent donc être décrites comme un ensemble de jeux didactiques, chacun ayant un enjeu particulier lié à la construction de savoirs. Chaque jeu comporte des joueurs représentant respectivement l'instance professeur $(P)$ et l'instance élève $(E)$ (Sensevy, 2007, p. 14). E gagne s'il produit la stratégie gagnante de son propre mouvement (proprio motu). P, qui connait la stratégie gagnante, gagne si E gagne. Il s'agit donc d'un jeu collaboratif, un jeu conjoint inclus dans une action conjointe (Sensevy, 2009, p. 115). Toutefois, l'action conjointe ne signifie pas pour autant que $E$ et $P$ aient les mêmes buts, si bien que des transactions à propos du savoir concerné sont inévitables au cours du jeu pour que celui-ci progresse. Pour décrire un jeu didactique, la théorie propose un ensemble de concepts que nous allons maintenant préciser.
Le triplet des genèses (mésogenèse, chronogenèse et topogenèse) rend compte d'un point de vue dynamique du contexte de l'action, de son objet et de la place qu'y prennent les différents acteurs. La mésogenèse concerne la genèse du milieu didactique. Selon Brousseau (2003, p. 3), le milieu didactique est « tout ce qui agit sur l'élève [ou l'enseignant] ${ }^{7}$ et tout ce sur quoi l'élève [ou l'enseignant] agit ». Sensevy l'envisage à la fois comme un contexte cognitif commun rendant possible l'action conjointe et comme un contexte antagoniste résistant aux actions et pensées des élèves (2007, p. 23). II inclut des éléments matériels et conceptuels amenés par les élèves ou le professeur au cours du jeu, mais aussi le résultat du processus sémiotique co-construit au cours des interactions. La mésogenèse décrit donc le processus par lequel le professeur et les élèves organisent ou réorganisent le milieu. La chronogenèse, c'est-à-dire la genèse du temps didactique, décrit l'évolution du savoir résultant des transactions successives entre les acteurs. La topogenèse, c'est-à-dire la genèse des positions dans la classe, décrit la répartition de la responsabilité épistémique entre professeur et élève.

Ces trois dynamiques évoluent de concert: "à chaque état de la mésogenèse correspond un état de la topogenèse et un état de la chronogenèse au regard des savoirs en jeu " (Amade-Escot \& Venturini, 2009, p. 29). Leur évolution est liée au contrat didactique qui peut être considéré comme un système essentiellement implicite d'attentes mutuelles entre professeur et élèves, d'habitudes conjointes ou encore d'attribution mutuelle d'intentions, à propos d'un savoir en jeu (Sensevy, 2009, p. 115). Ce dernier évoluant au cours du temps, le contrat évolue, donnant lieu à une série de ruptures, à l'initiative du professeur et/ou des élèves.

Pour initier et maintenir la relation didactique au cours du jeu, le professeur intervient de différentes manières et un quadruplet de descripteurs du jeu didactique en rend compte: définir, dévoluer, réguler, institutionnaliser (Sensevy, 2007, p. 29). Ainsi, le professeur définit le jeu didactique, introduisant les objets nécessaires et précisant ses règles. II dévolue le jeu, en agissant de telle manière que les élèves acceptent de s'engager dans le jeu et d'y jouer de manière adéquate. II s'agit là d'un processus qui se poursuit tout au long du jeu. II régule aussi le jeu, intervenant pour que les élèves modifient leur action et parviennent à gagner. Enfin, il institutionnalise le savoir en jeu, signifiant aux élèves qu'ils ont produit le savoir enjeu du jeu et que celui-ci est valide au-delà de la classe, dans une communauté beaucoup plus large.

L'action ainsi modélisée est associée à des déterminants, principalement pour le professeur, plus 
évoqués que réellement formalisés dans la TACD. Ainsi son action dans la classe serait déterminée à la fois:

- par son rapport personnel aux savoirs (Chevallard, 2003) qui sont en jeu dans les tâches proposées aux élèves (Sensevy, 2007, p. 36);

- par les différentes institutions auxquelles cette action est adressée; en effet, dans la plupart d'entre elles, l'enseignant ne peut pas apparaître très longtemps comme un mauvais sujet;

- par son épistémologie pratique. Celle-ci est « d'abord une théorie plus ou moins implicite de la connaissance (des savoirs enseignés), de son sens, de son usage, des relations que telle connaissance entretient avec telle autre " (Sensevy, 2007, p. 37). Constituant en quelque sorte l'arrière-plan des rapports précédents, " elle est aussi adressée, en tant qu'épistémologie des transactions didactiques, charriant ainsi une conception de ce qu'est l'apprentissage, de ce que peuvent être les difficultés d'apprentissage, de ce que peuvent signifier des différences entre élèves, etc. "

Cette théorie nous amène à préciser notre question de recherche. II s'agira pour nous de caractériser l'action conjointe professeur/élève dans le cas de la mise en œuvre d'une démarche d'investigation dans la classe: quelles dynamiques méso, chrono, topogénétiques peut-on voir à l'œuvre dans tout ou partie de la séance, en lien avec les modalités de définition, des processus de dévolution et d'institutionnalisation et les types de régulation des différents jeux? Quels déterminants pour le professeur peut-on inférer de ces dynamiques? Pour répondre à ces questions, nous avons développé une méthodologie spécifique que nous allons décrire.

\section{MÉTHODOLOGIE}

Les données que nous avons étudiées ont été mises à notre disposition dans le cadre du projet Vidéos de situations d'enseignement et d'apprentissage (projet ViSA $^{8}$ ) (Veillard \& Tiberghien, à paraître). Celui-ci a réuni des chercheurs en sciences de l'éducation utilisant pour leurs recherches des vidéos de classe et les données associées mises à disposition, via une base de données, par d'autres chercheurs pour en faire l'analyse avec leurs cadres et leurs problématiques (Venturini, 2010). Notre corpus a été recueilli par l'équipe de Serge Leblanc (LIRDEF, université Montpellier 2 et 3) pour des analyses spécifiques du type de celles développées par exemple par Leblanc, Ria et Veyrunes (à paraître). Nous décrivons tout d'abord ce corpus avant de préciser le traitement dont il a fait l'objet dans une perspective didactique, différente de celle pour laquelle il a été constitué à l'origine.

\section{Les données à disposition}

Plusieurs pièces font partie de notre corpus:

- la vidéo de la séance de classe: d'une durée de 55 minutes, elle a été réalisée en classe de $4^{\mathrm{e}}$ et concerne l'enseignement de la loi d'additivité des tensions dans un circuit série à partir d'une démarche d'investigation, menée par un jeune enseignant. Cette séance vient après une séance sur la mesure des tensions et sur la notion de tension nominale. La classe filmée comporte une vingtaine d'élèves en milieu rural et elle semble à la fois travailleuse et rompue à des activités du type de celles qui ont été menées:

- une vidéo de type autoconfrontation: pendant qu'il regarde la vidéo de sa séance de classe, l'enseignant, filmé, répond à des questions sur ce qu'il a réalisé ou commente certaines de ses actions, ce qui nous a permis de le considérer comme un entretien apportant certaines informations sur le déroulement de la séance;

- la fiche distribuée aux élèves, contenant les consignes et des espaces pour les réponses aux différentes activités proposées.

\section{Retranscription de la séance de classe, condensation de données, analyse a priori}

Après avoir identifié les exigences institutionnelles pour avoir une idée des enjeux de la séance et après l'avoir visionnée plusieurs fois pour nous l'approprier, toutes les interactions entre le professeur et les élèves ainsi que les gestes et attitudes des acteurs qui nous paraissaient significatives au regard du savoir en jeu ont été retranscrites.

Une analyse de ces données nous a conduits, en référence à la $T A C D$, à les structurer en jeux. Cette structuration a été d'abord conduite indépendamment par chacun d'entre nous. La discussion qui a suivi a permis après accord de formaliser neuf jeux d'apprentissage successifs. Leurs traits caractéristiques sont décrits dans le tableau 2 qui donne ainsi une vue d'ensemble de la séance. Les différents jeux ont été définis en considérant ce que l'élève doit réussir à faire pour gagner au jeu, c'est-à-dire en tenant compte à la fois de l'enjeu à satisfaire, de la règle à suivre pour y parvenir et du milieu supportant l'action didactique. Ce découpage nécessite aussi de choisir un niveau de granularité approprié pour 
percevoir les dynamiques en jeu dans une seule séance (soit pour nous des jeux durant de 3 à 18 minutes).

Cette phase a aussi permis d'identifier la tâche donnée aux élèves et d'en faire l'analyse a priori), en lien avec les aspects institutionnels et épistémologiques afin de prévoir une partie des comportements possibles des élèves. Enfin, l'entretien de type autoconfrontation a aussi été retranscrit et les informations qu'il comportait structurées de manière thématique ou en lien avec certaines parties de la séance.

\section{Analyse de la séance de classe}

La retranscription de la séance a été réalisée à l'aide du logiciel Transana ${ }^{9}$, spécialisé dans l'analyse de vidéos. Ce logiciel permet de définir, aussi bien a priori qu'en cours d'analyse, des catégories comportant chacune plusieurs mots-clés et d'affecter ces derniers à une partie de la vidéo pour la caractériser. Deux approches ont été mises en œuvre lors de cette caractérisation, nous présentons ici l'une d'entre elles, l'autre faisant l'objet d'un prochain article avec des résultats complémentaires. Ainsi, nous avons découpé la séance et plus particulièrement chacun des jeux qui la constituent en épisodes successifs. Chaque épisode (d'une durée moyenne inférieure à la minute) est caractérisé à l'aide des catégories suivantes et des mots-clés associés (voir annexe):

- le jeu auquel il appartient (jeu 1, jeu 2, etc.);

- la nature de l'activité menée durant l'épisode (activité liée à l'enseignement de l'électricité ou à la gestion matérielle de l'environnement de travail);

- l'organisation de l'activité durant l'épisode (organisation collective, en binôme, mixte);

- les thèmes traités dans la séance (comme la mesure de tension, ou la loi des tensions);

- les catégories en lien avec le cadre théorique: mésogenèse (introduction d'un objet dans le milieu, reprise neutre d'un objet introduit par un élève, etc.); topogenèse (sous la responsabilité du professeur, des élèves, ou mixte); chronogenèse en lien avec le rythme de progression du savoir (arrêt, avancée, ralentissement, etc.); techniques didactiques (définition, dévolution, régulation, etc.).

Deux épisodes successifs se distinguent donc par au moins un mot-clé différent. Les catégories et les motsclés ont été déterminés à la fois a priori à la suite de travaux précédents (par exemple Tiberghien, Malkoun \& Seck, 2008) et de manière inductive à partir de l'analyse de chaque épisode. Cette indexation et le découpage associé en épisode nécessitent à la fois une vision locale au niveau micro des interactions et une perception plus globale au niveau mésoscopique (intermédiaire) du jeu, qui comporte plusieurs épisodes articulés. Les allers et retours entre ces deux niveaux sont permanents au cours de ces opérations. Ces indexations sont réalisées à partir de l'analyse didactique de l'action (principalement discursive mais aussi gestuelle), analyse qui est donc centrée sur les savoirs et leur évolution. En considérant chaque jeu dans son ensemble, on peut voir les évolutions dans les indexations d'un épisode à l'autre et caractériser ainsi les dynamiques liées aux trois genèses (voir par exemple le premier jeu analysé).

Une fois l'indexation réalisée, Transana donne une vision de tout ou partie de la séance à partir de la répartition des différents mots-clés (pourcentage d'épisodes caractérisés par un mot-clé ou pourcentage temporel correspondant). II offre aussi une vision plus dynamique, à partir de graphiques représentant l'évolution des mots-clés au cours du temps, ce qui permet de dégager certaines spécificités des jeux analysés (voir figure 2 et suivantes). En combinant ces résultats à une analyse systématique des interactions didactiques fondée sur l'avancée des savoirs dans les différents jeux, nous cherchons à décrire la dynamique des transactions entre les acteurs à propos des savoirs en jeu. Nous avons spécifiquement documenté les phases collectives dans la mesure où les interventions de l'enseignant relevant de la direction d'étude étaient systématiquement assurées collectivement par arrêt du travail de tous les binômes. Cette caractérisation de la séance permet d'inférer un certain nombre de ses déterminants mis en regard avec les informations apportées par l'enseignant dans son entretien de type autoconfrontation, lorsqu'elles sont disponibles.

Analysons maintenant a priori la séance pour se faire une idée des comportements possibles des élèves et des difficultés qu'ils vont rencontrer avant de présenter le synopsis de la séance effectivement réalisée.

\section{ANALYSE A PRIORI ET SYNOPSIS DE LA SÉANCE ANALYSÉE}

\section{Analyse a priori}

L'analyse apriori de la séance a été réalisée à partir du document distribué aux élèves, en prenant appui sur le programme officiel, sur les savoirs de référence et sur des résultats des recherches en didactique de la physique. Le document distribué décrit le problème à résoudre par les élèves (figure 1) ainsi que les activités qu'ils ont à réaliser pour y parvenir. Le tableau 1 permet 
Tableau 1. Parallèle entre la démarche du professeur observé et la démarche d'investigation proposée dans les instructions officielles

\begin{tabular}{|c|c|}
\hline Démarche du professeur observé & $\begin{array}{l}\text { Démarche d'investigation proposée } \\
\text { dans les instructions officielles }\end{array}$ \\
\hline Proposition d'une « situation problème » & Choix d'une situation problème \\
\hline «Reformuler la question » après le débat collectif & $\begin{array}{l}\text { Appropriation du problème par les élèves } \\
\text { (et reformulation de la question) }\end{array}$ \\
\hline $\begin{array}{l}\text { "Réfléchir : dessiner le montage électrique à } \\
\text { réaliser et [proposer] les diverses mesures à } \\
\text { effectuer " }\end{array}$ & $\begin{array}{l}\text { Formulation d'hypothèses explicatives et de } \\
\text { protocoles possibles }\end{array}$ \\
\hline \multirow[t]{2}{*}{ «Expérimenter » et remplir le tableau de mesures } & $\begin{array}{l}\text { Investigation ou résolution du problème conduite par } \\
\text { les élèves }\end{array}$ \\
\hline & Échange argumenté autour des solutions élaborées \\
\hline $\begin{array}{l}\text { «Exploiter le résultat des mesures » afin de } \\
\text { donner « la relation entre la tension aux bornes } \\
\text { d'un générateur et les tensions aux bornes des } \\
\text { récepteurs qui constituent un circuit en série » } \\
\text { et «proposer à Pierre une solution pour que son } \\
\text { montage fonctionne » }\end{array}$ & Acquisitions et structuration des connaissances \\
\hline «Utiliser les connaissances » & Opérationnalisation des connaissances \\
\hline
\end{tabular}

Note : les éléments entre guillemets renvoient directement au contenu de la fiche élève.

de comparer cette liste d'activités avec les différentes étapes de la démarche d'investigation (MEN, 2008).

La lecture du tableau nous permet tout d'abord d'identifier l'objectif de l'enseignant dans cette séance à travers la relation qu'il veut faire établir aux élèves. II s'agit pour lui d'enseigner la loi d'additivité des tensions dans un circuit série en la faisant établir à partir de mesures. En cela, il est en conformité avec les exigences institutionnelles (MEN, 2008, p. 19). Par ailleurs, même si la lecture du tableau montre aussi un assez large recouvrement entre les deux démarches, deux points attirent notre attention à propos de la démarche de l'enseignant observé. Le premier concerne l'absence dans la fiche, d'une phase de formalisation explicite des hypothèses explicatives précédant la conception du protocole expérimental. Cela nous amène à penser que les éléments qui auraient un statut d'hypothèse dans la séance seront moins valorisés que les autres. Le second point concerne l'absence d'un temps d'« échange argumenté " autour des résultats, mais cette absence peut apparaître plus légitime dans la mesure où ce temps d'échange serait oral et n'a pas forcément vocation à laisser des traces sur la fiche.

Analysons maintenant le problème proposé aux élèves (figure 1), situation qualifiée de "déclenchante " dans les textes officiels.

Si l'on peut penser que les élèves et en particulier les garçons sont susceptibles de se sentir concernés en s'identifiant au personnage de l'histoire, la situation apparaît complexe sur le plan didactique et physique. Selon les élèves et le guidage de l'enseignant, on peut imaginer un éventail de réponses allant de la réponse quasi intuitive mais éloignée de la loi d'additivité des tensions (si les ampoules «brillent de manière inégale et plutôt faiblement »- et en réalité pas du tout -, il suffit d'enlever des ampoules ou de rajouter une pile pour qu'elles brillent mieux), à une réponse beaucoup plus sophistiquée que nous détaillons maintenant. En utilisant le modèle de la tension nominale qu'ils connaissent, les élèves peuvent prévoir que les ampoules brillent faiblement parce que la tension à leurs bornes est inférieure à leur tension nominale. Ils 
Pierre vient de fabriquer une très belle cabane au fond des bois.

Bien décidé à y passer une nuit, il décide de fabriquer un système d'éclairage à partir de matériels récupérés dans le garage de son père :

- Une pile toute neuve sur laquelle est inscrite la tension $12 \mathrm{~V}$;

- Diverses ampoules de torche électrique symbolisées ci-contre.

- Un interrupteur et des fils de connections.

$\mathrm{Ce}$ dernier assemble en série les 10 ampoules et s'empresse de tester son montage, mais sa déception est grande car les lampes brillent de manière inégale et plutôt faiblement.

Peux-tu l'aider à résoudre son problème?

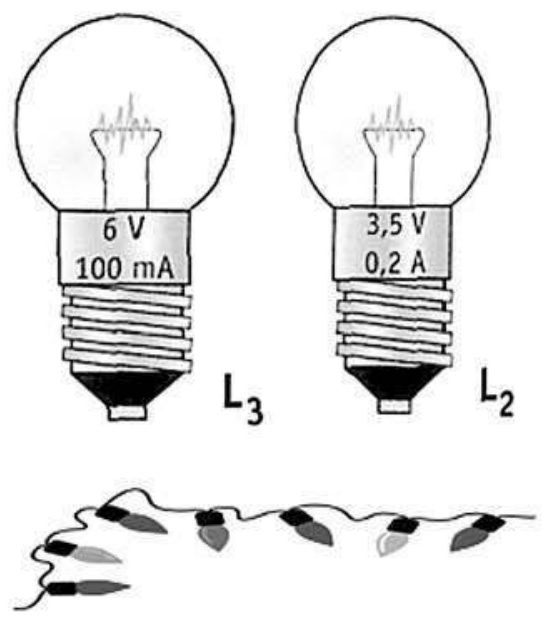

pourraient éventuellement le vérifier en reconstituant en classe une situation analogue à celle proposée. En s'appuyant sur l'expérience acquise dans les classes précédentes au cours desquelles ils ont branché une, deux, voire trois ampoules en série avec un générateur, les élèves pourraient alors suggérer de supprimer des ampoules ou de remplacer la pile par une pile à tension plus élevée pour augmenter la tension aux bornes de chaque ampoule et atteindre ainsi sa tension nominale. L'hypothèse implicite la plus simple sous-tendant cette proposition est l'additivité des tensions dans le circuit série et celle-ci pourrait devenir explicite avec l'aide de l'enseignant. II s'agirait alors pour les élèves de proposer un circuit et une procédure associée pour étudier cette hypothèse. Une fois validée expérimentalement, l'enseignant pourrait ensuite l'institutionnaliser comme loi en se référant aux connaissances de la physique. Dans un dernier temps, l'exploitation de cette loi combinée à la notion de tension nominale amènera alors les élèves à résoudre le problème en concluant qu'il faudrait que, dans le circuit de la cabane, la somme des tensions nominales des ampoules corresponde à la tension du générateur qui les alimente.

On obtient donc là un ensemble complexe comprenant successivement:

- l'usage d'un modèle pour effectuer une prévision;

- l'émission d'une hypothèse en termes de tension sur le fonctionnement d'un circuit série;

- la validation de cette hypothèse;
- l'établissement d'une loi (l'enjeu de savoir de la séance) après institutionnalisation;

- une combinaison de la prévision d'un modèle et de l'application de la loi obtenue pour résoudre le problème de départ.

Toutefois, si l'on reproduit là le raisonnement que les élèves de $4^{\mathrm{e}}$ pourraient faire pour résoudre le problème, il faut rajouter qu'il est scientifiquement insuffisant. II faudrait en effet aussi tenir compte des intensités nominales des deux séries d'ampoules qui, de plus, sont différentes (voir figure 1); ceci rend impossible que toutes les ampoules éclairent normalement en même temps. De plus, on remarque que l'aspect hypothétique a dans ce processus une importance qui ne semble pas apparaître dans la fiche, comme nous l'avons pointé précédemment.

Pour terminer, on sait que le concept de tension n'est pas sans causer des difficultés aux élèves (Benseghir, 1988; Closset, 1989). Ici toutefois, on peut penser qu'elles ne gêneront pas la résolution du problème. En effet, la tension est dans le programme de $4^{\mathrm{e}}$ ce qui "se mesure avec un voltmètre branché en dérivation à ses bornes " (MEN, 2008, p. 19) et pour les élèves généralement "ce qui est aux bornes de la pile " (et qu'on « retrouve » aux bornes des ampoules). De plus, et probablement pour les mêmes raisons, la loi d'additivité des tensions ne semble pas poser des problèmes aux élèves (Allevard, Carron, Martin et al., 1991) au moins tant 
Tableau 2. Le synopsis de la séance

\begin{tabular}{|c|c|}
\hline Chrono & Descriptif des différents jeux constituant la séance ( $P$ = Professeur ; J = Jeu) \\
\hline $1: 26$ & Installation des élèves. \\
\hline $7: 35$ & $\begin{array}{l}\text { J3. Proposer une solution/explication pour le problème } \\
\text { P demande aux élèves de proposer des « solutions » puis des « explications » à propos de cette situation. Les élèves } \\
\text { proposent d'ajouter une pile, d'enlever des ampoules. Pour une élève « si on ajoute tous les volts des lampes, c'est } \\
\text { trop pour la pile ". Ces éléments sont notés au tableau sans commentaire. P distribue une fiche rappelant le problème, } \\
\text { proposant les activités qui vont suivre et des questions pour leur interprétation. }\end{array}$ \\
\hline 29:58 & $\begin{array}{l}\text { J5. Proposer le schéma d'un dispositif expérimental permettant, à partir de mesures, de répondre à la question } \\
\text { "comment se répartissent les tensions" } \\
\text { Les élèves schématisent par binôme un circuit conçu à partir de deux lampes différentes, deux générateurs et un } \\
\text { multimètre et susceptible de répondre à la question « comment se répartissent les tensions dans le circuit série ». } \\
\text { retient le dernier de trois circuits qu'il a choisi de faire présenter lors de la mise en commun, puis décrit la procédure que } \\
\text { les élèves devront suivre (réaliser un circuit série avec deux ampoules et un générateur, mesurer les tensions aux } \\
\text { bornes de chaque dipôle, puis refaire la même chose dans un second circuit en changeant de générateur) et enfin, } \\
\text { donne le tableau de mesures à remplir. }\end{array}$ \\
\hline $51: 30$ & $\begin{array}{l}\text { J7. Comparer les tensions aux bornes des deux ampoules à celle du générateur } \\
\mathrm{P} \text { demande à un élève de répondre à la première question de la fiche, visant à « comparer la tension aux bornes de } \\
\text { chaque ampoule à celle du générateur ». Alors que l'élève interrogé compare entre les deux circuits les tensions aux } \\
\text { bornes des ampoules, } P \text { demande de comparer les tensions des ampoules au sein d'un même circuit et rattache leur } \\
\text { différence à une différence de tension nominale. }\end{array}$ \\
\hline $54: 48$ & $\begin{array}{l}\text { J8. Proposer une relation mathématique entre les tensions des différents dipôles du circuit série (loi des } \\
\text { tensions dans un circuit série) } \\
\text { Un élève interrogé et aidé par } \mathrm{P} \text { avance l'idée que la somme des tensions aux bornes des deux ampoules est à peu } \\
\text { près égale à la tension aux bornes du générateur alors que la cloche marque la fin de la séance. P prend en charge } \\
\text { la formalisation de cette relation en langue naturelle. }\end{array}$ \\
\hline 56:01 & $\begin{array}{l}\text { J9. Appliquer la loi des tensions au problème de départ } \\
\text { P demande aux élèves en quoi la conclusion précédente règle le problème de départ. Ceux-ci faisant état des solutions } \\
\text { déjà proposées dans le jeu } 3, P \text { avance lui-même sa conclusion : « il faut que la somme des tensions nominales des } \\
\text { ampoules soit équivalente à la tension du générateur ». }\end{array}$ \\
\hline $57: 00$ & quittent la salle. \\
\hline
\end{tabular}


qu'elle est la seule grandeur à être en jeu, ce qui est le cas ici (l'intensité n'est pas mentionnée).

\section{Synopsis de la séance}

Le tableau 2 présente successivement les neuf jeux tels que nous les avons découpés dans le flot de l'action. Leur dénomination correspond à l'activité que les élèves sont censés réussir pour « gagner au jeu » correspondant. Elle est fondée sur le sens donné au jeu par l'enseignant, qui peut être différent de celui donné par les élèves. C'est ainsi en cherchant à identifier les enjeux, les règles, les stratégies, les changements importants dans le milieu et le contrat que nous sommes parvenus au découpage proposé dans le tableau 2. On peut déjà remarquer à la lecture de ce tableau que la trame des activités proposées dans la fiche distribuée aux élèves a été suivie par l'enseignant, à l'exclusion du réinvestissement des connaissances, qui n'a pas été mené durant la séance faute de temps. Toutefois, certaines activités ont donné lieu à plusieurs jeux.

\section{RÉSULTATS}

Examinons maintenant les résultats issus de l'analyse de la séance. Après avoir donné quelques caractéristiques générales de la séance, nous examinerons successivement les jeux 3 et 4 qui conduisent à la formulation d'une question à résoudre, les jeux 5 à 8 au cours desquels la résolution de la question posée conduit à la loi d'additivité des tensions, et enfin le jeu 9 au cours duquel l'enseignant revient sur la situation de départ.

\section{Quelques caractéristiques générales de la séance: élèves au travail, contrat familier, pression temporelle sur les acteurs}

Notre analyse fait apparaitre 88 épisodes en 57 minutes de séance. Soixante d'entre eux sont dédiés au travail en électricité et occupent pratiquement 45 minutes soit les trois quarts du temps. Le temps restant (12 minutes) est réservé à la gestion de l'environnement de travail. Aucun rappel à l'ordre ou au travail n'a été adressé aux élèves.

Les activités sont plutôt collectives ou mixtes (l'enseignant intervient alors pour toute la classe pendant que les élèves travaillent en binôme) : celles-ci occupent en effet 41 épisodes sur 60 et pratiquement 25 minutes sur 45. Les 20 minutes restantes (un peu moins d'un tiers de la séance totale) sont consacrées aux trois activités en binôme (formuler une question, concevoir un circuit, réaliser le circuit et faire les mesures), la quatrième (exploiter les mesures) ayant finalement été menée collectivement faute de temps.

Le travail en binôme s'effectue sous une pression temporelle constante. Ainsi, quatre interventions de l'enseignant ont été faites en huit minutes sur ce thème pendant le jeu 5 où les élèves conçoivent un circuit (par exemple « je vous donne deux ou trois minutes pour faire ça » (min. 14:37); "vous avez deux ou trois minutes encore » (min. 20:49), et il y en a autant pendant les 16 minutes où les élèves le réalisent. Mais la pression temporelle existe aussi sur l'enseignant dans le fonctionnement collectif, ce qu'il confirme au cours de son entretien («pendant tout le TP, [ma préoccupation] c'était la gestion du temps, je voulais avancer absolument, absolument »).

Enfin, les éléments pérennes du contrat didactique semblent stabilisés; comme nous l'avons dit plus haut, les élèves ont l'habitude du fonctionnement qui leur est proposé: « vous avez l'habitude du scénario, je vous le projette au tableau je vous explique et rapidement on va mettre en place un débat » (min. 03:42). L'enseignant le confirme d'ailleurs dans son entretien: « ils sont habitués, ils savent le faire... c'est la première année que je les ai donc on a mis cela en place en début d'année, ils ne le faisaient pas avant. »

\section{De la situation problème à la formulation de la question à résoudre expérimentalement: un chemin difficile pour tous les acteurs}

L'analyse a priori a montré que la situation proposée était à la fois complexe et éloignée de l'enjeu de savoir. En effet, l'additivité des tensions dans un circuit série ne découle pas directement de la situation proposée, elle ne peut être émise comme hypothèse qu'à la suite d'un raisonnement, et elle n'est pas la seule à intervenir pour résoudre les dysfonctionnements du circuit proposé. L'analyse des jeux 3 et 4 permet de comprendre comme l'enseignant a géré cette situation.

L'analyse de la figure 2 proposant l'évolution des mots-clés au cours des différents épisodes du jeu 3 fait apparaître une dynamique particulière. Alors que le processus de dévolution s'opère (ligne 9 du graphe), que les élèves assument la responsabilité de faire avancer le savoir (ligne 8) et que celui-ci avance (ligne 7), l'enseignant semble sur la réserve: il propose une première régulation (ligne 10) au cours de laquelle il précise la règle du jeu (ligne 4) et il se contente de reprendre de manière neutre 
Figure 2. Extrait du graphe construit par le logiciel Transana montrant l'évolution de certains mots-clés au cours du jeu 3 (Proposer une solution/explication pour le problème $-\min$. 6:07 à 8:35). Chaque bloc correspond à un épisode

01 Jeux: J3 Proposer solution explication
02 Objet épisode : Electricité
03 Organisation Activité : Collective
04 Mesogenèse : Préciser règle du jeu
05 Mesogenèse : Reprise neutre d'un objet introduit par élève
06 Chronogenèse : Arết
07 Chronogenèse : Avancée
08 Topogenèse : Elève
09 Techniques didactiques : Devoluer processus
10 Techniques didactiques : Réguler

\section{Extrait 1. Interactions du jeu 3 ( $\min .6: 13$ à 7:35 - $\mathbf{P}=$ professeur, $E 1=$ élève). Les éléments en gras sont des éléments significatifs pointés dans l'analyse}

1. P: moi je vous pose des questions. Est-ce que franchement vous avez une idée de ce qui va (inaudible.)? Je ne vous demande pas la solution tout de suite mais... je vous demande d'amener une solution, je vous demande d'amener un élément de réponse. Alors t'as une petite idée? (Des élèves lèvent le doigt) Bon je note vos idées ici hé ( $P$ se dirige vers le tableau). (0:06:21.4)

2. E1 : en fait je pense que la pile de $\mathbf{1 2}$ volts elle est pas assez puissante pour euh...

3. $\quad \mathrm{P}$ : (En écrivant au tableau) la pile est pas assez puissante.

4. E1: pour allumer toutes les lampes c'est pour ça qu'elles sont faibles.

5. $\mathrm{P}$ : d'accord c'est bien faudra expliquer pourquoi la pile est pas assez puissante pas assez forte. Julian t’as une idée? (0:06:40.7)

6. E1: ça pourrait se solutionner avec deux piles de 12 volts.

7. $\quad \mathrm{P}$ : alors là vous me proposez des solutions. Je vous demande pas une solution tout de suite je vous demande d'abord une explication, essayez de comprendre ce qu'il se passe encore pour résoudre le problème effectivement. Mathieu? (0:06:53.2)

8. E1: trop d'ampoules.

9. $\mathrm{P}$ : trop d'ampoules d'accord ça peut être des éléments de réponse effectivement? (Pécrit au tableau) D'autres suggestions? (0:07:02.4)

10. $\mathrm{P}$ : Emma? (elle lève le doigt)

11. E1: si on ajoute tous les volts peut-être c'est trop pour euh, les 12 volts de la pile?

12. $\mathrm{P}:$ si on ajoute tous les volts de quoi?

13. E1: de l'ampou enfin des lampes!

14. P: des lampes d'accord.

15. P: (En écrivant en haut du tableau) si on ajoute tous les volts c'est ça que tu m'as dit?

16. E1: oui des lampes. (0:07:19.0)

17. P: tout le monde comprend ce que j'écris au tableau là? II y a pas de soucis vous êtes d'accord avec vos camarades?

18. E1: oui. (0:07:27.0)

19. $\mathrm{P}$ : si on ajoute tous les volts des ampoules c'est ça que tu m'as dit c'est supérieur à la pile hé?

20. E1: oui (P finit de copier l'idée au tableau). (0:07:35.0) 
Figure 3. Extrait du graphe construit par le logiciel Transana montrant l'évolution de certains mots-clés au cours du jeu 4 (Formuler la question à résoudre expérimentalement $-\min$. 7:35 à 13:40)

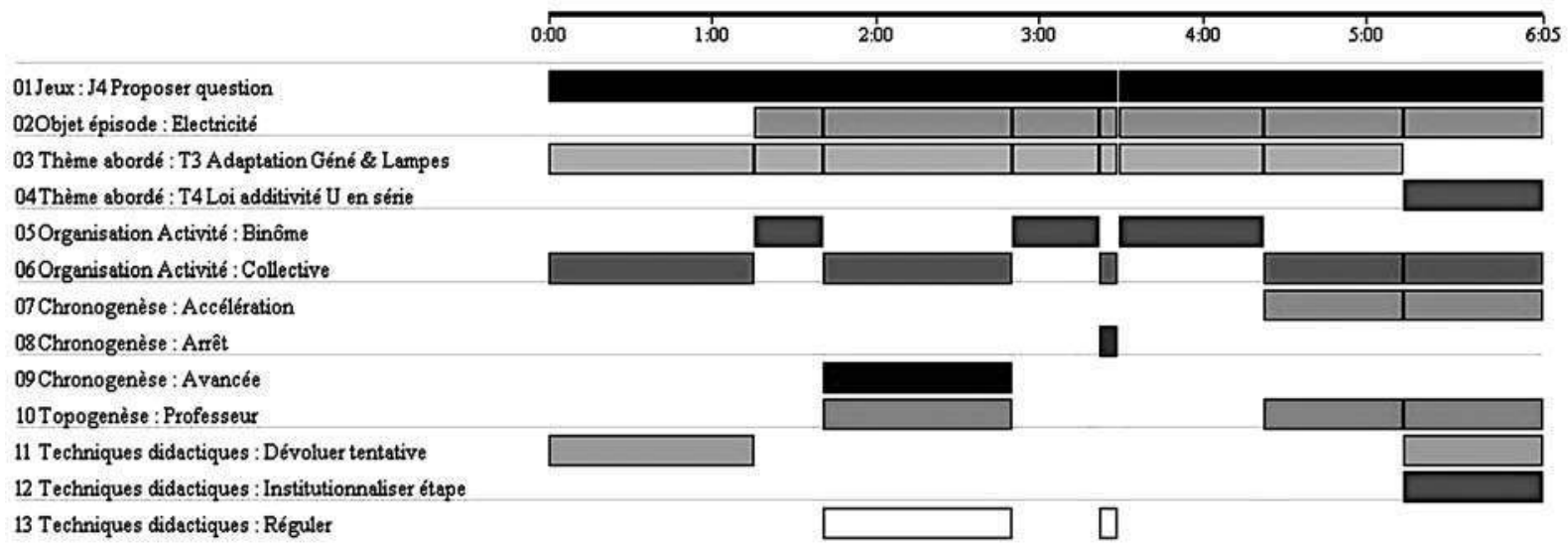

les propositions des élèves (ligne 5). L'examen de la retranscription de ce jeu proposée dans l'extrait 1 permet de donner du sens à la dynamique observée.

Le jeu est d'abord centré sur la solution du problème exposé comme le montre le tour de parole 1 (TDP 1): « je vous demande d'amener une solution, je vous demande d'amener un élément de réponse ". La dévolution s'opère fortement sur les aspects pratiques, et les élèves introduisent dans le milieu des solutions concrètes et immédiates comme l'analyse a priori le laissait supposer: ajouter des piles, mettre une pile plus puissante, enlever des ampoules (TDP 2, 4, 6, 8). Cela amène le professeur à reformuler la règle du jeu qu'il voudrait jouer avec ses élèves, " alors là vous me proposez des solutions. Je vous demande pas une solution tout de suite je vous demande d'abord une explication, essayez de comprendre ce qu'il se passe encore pour résoudre le problème effectivement » (TDP 7). Peut-être en réponse à cette régulation, Emma introduit dans le milieu une explication hypothétique (avancée de la chronogenèse) qui, exploitée, pourrait être féconde puisqu'elle sousentend implicitement que les tensions s'additionnent dans un circuit série: "si on ajoute tous les volts [des lampes] peut-être c'est trop pour euh, les 12 volts de la pile » (TDP 11). L'enseignant se contente de noter de manière neutre au tableau cette proposition comme les précédentes. Ce sont donc uniquement les élèves qui assument la responsabilité de l'avancée des savoirs dans ce jeu (topogenèse). De par la neutralité de l'enseignant, tous les objets introduits dans le milieu ont le même statut (mésogenèse). Pourtant, la proposition faite au TDP 11 est différente, de nature beaucoup plus conceptuelle, alors que les autres sont relatives aux propriétés des composants et aux actions dont ils peuvent faire l'objet. Cela clôt le jeu et la phase d'échange collectif.

Le jeu 4 est plus clairement défini par l'enseignant: " vous avez à reformuler la question, quel est pour vous le problème, d'accord? » (min. 7:38), en sachant que « vous avez des éléments de réponse au tableau si vraiment vous n'avez pas compris » (min. 8:30). Sa dynamique est visualisée sur la figure 3 .

On constate que l'activité censée se dérouler en binôme est finalement majoritairement menée collectivement en classe entière (lignes 5 et 6 ). On constate aussi que la dévolution tentée (ligne 11) ne s'opère pas: dans les phases collectives qui suivent les phases en binôme, la topogenèse est sous la responsabilité du professeur (ligne 10). Celui-ci n'exploite donc pas le travail que les élèves viennent de faire en binôme pour faire avancer le savoir (ligne 7 et 9 ). Certains groupes sont d'ailleurs toujours sur les aspects pratiques comme en témoigne la seconde régulation opérée (ligne 13): « attention pour l'instant, on essaie de comprendre le problème, on n'essaie pas de trouver une solution " (min. 11:04). II reste donc à examiner la nature des interventions de l'enseignant dans les trois épisodes collectifs au cours desquels le savoir avance. Moins de trente secondes après le début du travail en binôme, l'enseignant (" qui entend les chuchotements des élèves [...] et veut absolument les amener là-dessus » - extrait de 


\section{Extrait 2. L'enseignant introduit le fait que les ampoules du problème ne sont pas alimentées avec leur tension nominale (jeu 4 - min. 9:16 à 9:55)}

1. $\quad P$ : il y a peut-être un truc vous n'avez pas... On n'en a pas parlé. Les ampoules elles brillent mal. On l'a vu, ça, hier. Les ampoules elles brillent mal, ça veut dire quoi pour vous que les ampoules brillent mal? Je sais pas. François ça veut dire quoi que les ampoules brillent mal? Pour toi qu'est-ce que ça veut dire?

2. François: qu'elles éclairent pas.

3. P: (avant même que François n'ait terminé) oui d'accord. Et d'un point de vue heu... On s'est demandé pourquoi nous on l'a vu, qu'il peut y avoir des points de vue physiques scientifiques ça veut dire quoi?

4. E1: les volts sont pas adaptés.

5. P: les volts sont pas adaptés. Comment on pourrait dire ça autrement? Vous voulez parler des volts pour nous c'est?

6. E1: la tension.

7. P: la tension peut-être n'est pas la bonne d'accord? C'est pas la tension nominale qu'il y a aux bornes de chaque lampe. Donc dans vos questions c'est peut-être une question qu'on peut se poser ça! (P rajoute au tableau cette idée)

\section{Extrait 3. L'enseignant après avoir introduit l'idée de mesurer les tensions propose d'examiner leur “ répartition " dans le circuit série (jeu 4 - min. 13:30 à 13:40)}

1. $P$ : voilà on pourrait essayer de mesurer, vous êtes d'accord avec moi?

2. El: oui.

3. $\mathrm{P}$ : donc on veut savoir comment se répartit? (il écrit ce qu'il dit en même temps au tableau)

4. El: la tension.

5. P: voilà, la tension dans le circuit? On est d'accord ou pas ?

l'entretien) intervient en classe entière pour rappeler le modèle de la tension nominale (première étape du raisonnement évoqué dans l'analyse a priori). II en profite pour suggérer en même temps que ce serait une bonne question (voir extrait 2).

En limitant leur activité à un jeu d'écriture, les élèves sont alors en mesure de proposer une question liée à cette suggestion, si bien qu'en interrogeant un élève dont il vient de lire la proposition " pertinente " (extrait de l'entretien), le professeur peut écrire au tableau la question correspondante: "pourquoi la tension aux bornes des lampes n'est pas la bonne? » (min. 12:48). L'enseignement opère alors deux autres glissements successifs dans le jeu pour parvenir à ses fins. Le premier est opéré par effet de contrat: « et à la limite qu'est-ce qu'on pourrait se poser comme question? Si c'est pas la bonne [tension], c'en est une autre non... ? Qu'est-ce qu'on pourrait essayer de faire avec vos connaissances à vous? » (min 13:20). Deux éléments ayant fait l'objet de rappels en début de séance, le premier (tension nominale) ayant déjà été utilisé, la seule possibilité de réponse ne peut être liée qu'à la mesure de tension. Aussi, Yann, interrogé nommément, propose alors de « vérifier avec un voltmètre » (min. 13:30). Cette réponse n'est donc en rien motivée par les contraintes du problème ou par celles du savoir en jeu, elle est liée formellement au contexte précédent l'activité. Aussitôt, l'enseignant opère alors lui-même un dernier glissement dans le jeu vers la « répartition des tensions » (voir extrait 3).

Ainsi, à l'initiative du professeur et sans la participation réelle des élèves, on est passé rapidement d'une relation entre des objets (adaptation entre pile et ampoules) à une question mettant en jeu un concept physique, la tension, sa mesure et sa "répartition " entre différents dipôles (figure 3 , ligne 3 et 4), qui implique une vision systémique du circuit. On comprend bien qu'il s'agit là d'une réelle accélération de la chronogenèse provoquant une forte discontinuité dans les savoirs (ligne 7), et faisant de fait l'objet d'une institutionnalisation partielle (ligne 12). C'est sur elle que vont s'appuyer les quatre jeux suivants au cours desquels le problème de départ est alors abandonné. Ces quatre jeux concernent la 


\section{Extrait 4. Définition du jeu 5 (min. 13:40 à 14:28)}

1. P: on va attaquer la suite. Alors on vient de faire le 1) je reformule pour (inaudible), poser un petit peu, d'accord, vous vous rappelez? On a dit: voilà, quelle est la tension dans le circuit? Comment elle se répartit? Eh bien 2) je réfléchis, j'émets des hypothèses! Donc ce qu'on vous demande maintenant, c'est la deuxième partie de votre manipulation. Je demande maintenant sur transparent comme d'habitude eh ben de justifier un petit peu tout ça, de m'expliquer un petit peu tout ça. Vous m'avez dit: la tension se répartit de manière différente dans un circuit. D'accord! J'ai entendu dire: si on ajoute les volts des ampoules, d'accord ça fait ça dépasse les volts des?

2. E1: (murmure) de la pile.

3. P: de la pile. Ben tout ça, ça serait bien qu'on le re-vérifie. Donc pour cela je vous demande, c'est écrit hein, je vous demande un petit schéma électrique et des mesures à effectuer. Qu'est-ce qui vous serait utile pour justifier votre réponse? On est bien d'accord là-dessus? C'est clair pour tout le monde ?

\section{Extrait 5. Un binôme fait sa proposition de circuit et de mesures (min. 26:40 à 27:23)}

1. E1: on va faire le circuit en série avec deux lampes et un générateur.

2. P: oui.

3. El: puis on va brancher le voltmètre en dérivation.

4. P: oui?

5. E1: il mesure la tension et puis on va le faire deux fois, une fois avec chaque ampoule et puis après (explication inaudible) on verra si la pile est pas assez forte.

6. P: d'accord? Donc ça c'est pour tester si c'est la pile qui est pas assez forte d'accord?

7. E1: c'est pour tester si la pile elle est pas assez forte.

8. P: et tes mesures de tension c'est pour tester comment se répartit la tension dans le circuit.

9. E1: dans le circuit.

10. $\mathrm{P}$ : on est d'accord tout le monde là ?

conception du circuit et des mesures à réaliser (J5), la réalisation du circuit et des mesures (J6) et l'exploitation de ces mesures (J7 et J8) qui aboutit à la loi d'additivité des tensions.

De la question à résoudre sur la répartition des tensions à la loi d'additivité: plus d'espace pour les élèves, mais uniquement sur des savoirs techniques

Le jeu 5 est clairement défini, comme le montre l'extrait 4 (notamment au TDP 3), même si cette définition appelle quelques commentaires.

En effet, l'enseignant propose en abordant le point 2 de la fiche de réfléchir et d'émettre " des hypothèses ". C'est la première fois qu'il prononce ce terme qui ne figure pas dans la fiche élève. Ces hypothèses, si on suit ses propos, semblent relatives à "la tension [qui] se répartit de manière différente " et à l'explication d'Emma déjà commentée dans le jeu 3 (somme des tensions nominales des ampoules peut-être supérieure à la tension de la pile). On peut constater que l'enseignant les attribue toutes deux aux élèves alors que seule la seconde provient d'eux. Par ailleurs, une ambiguïté entre la situation de départ et le circuit d'étude semble s'installer chez certains élèves obligeant le professeur à repréciser l'objectif du circuit: faire des mesures pour voir comment se répartit la tension (voir extrait 5, TDP 6 et 8).

Cet extrait montre aussi que les élèves ont joué le jeu demandé, ce qui est confirmé par le fait que trois groupes soient passés au tableau en fin de jeu pour proposer circuit et mesures. Du reste, comme on peut le voir sur la figure 4, les modalités de travail en collectif et en binôme s'équilibrent dans le jeu quand il est question d'électricité (lignes 1 à 4 ; collectif: 6 minutes 24 ; binôme: 5 minutes 10; mixte: 1 minute 49). De plus, après le travail en binôme, les élèves sont en mesure de faire des propositions (lignes 5 à 9), assument ainsi la responsabilité de faire avancer le savoir (lignes 13 et 14) et le font effectivement avancer (lignes 10 et 12): dans les 6 minutes 18 terminant le jeu, la durée des 
Figure 4. Extrait du graphe montrant l'évolution de certains mots-clés au cours du jeu 5

(Proposer le schéma d'un dispositif expérimental permettant, à partir de mesures, de répondre à la question "comment se répartissent les tensions" - min. 13:40 à 29:58)

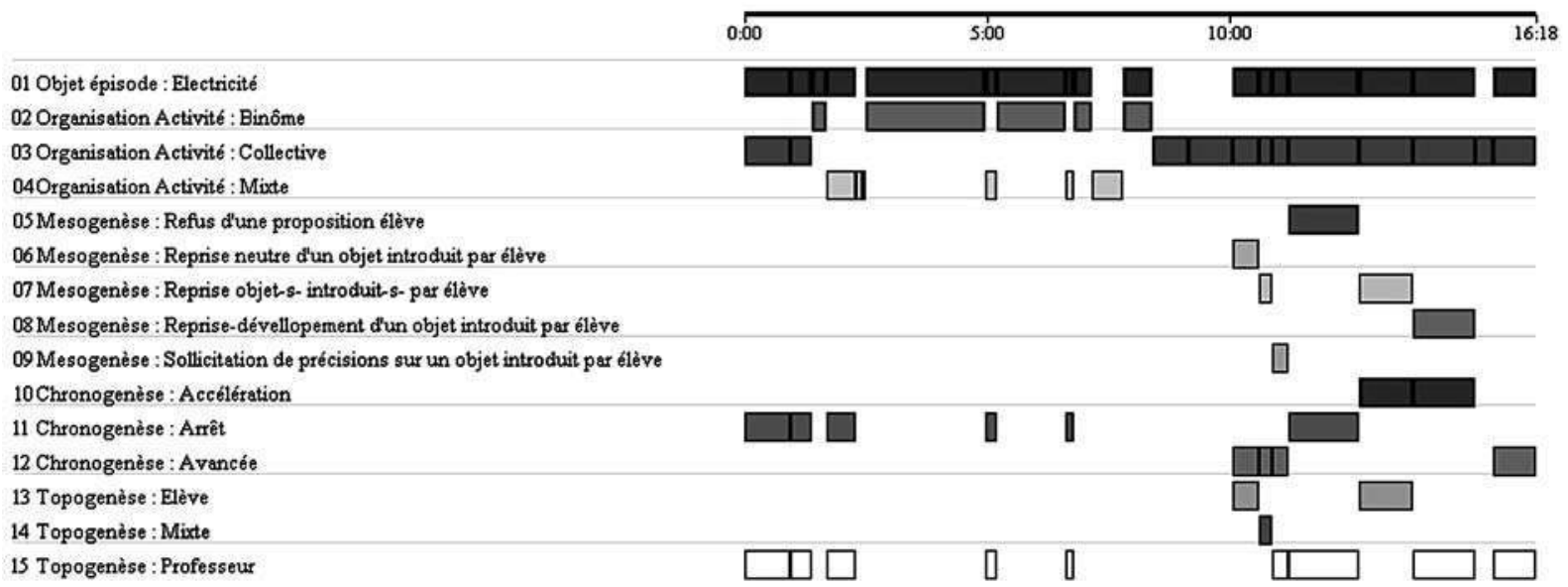

épisodes où la topogenèse est du côté des élèves ou est partagée entre professeur et élèves est significative par rapport à la durée des épisodes où le professeur a la responsabilité de faire avancer le savoir (1 minute 56 contre 4 minutes 24). Enfin, dans cette mise en commun, le savoir en jeu est principalement lié à des actions de montage et de mesure. L'institutionnalisation d'étape de l'enseignant reprend le circuit à réaliser ainsi que les mesures à faire et à disposer dans un tableau.

Le jeu 6 qui suit concerne la réalisation du circuit et la mesure des tensions aux bornes de deux ampoules L1 et L2 branchées en série alimentées tour à tour par un générateur de $4,5 \mathrm{~V}$ et de $9 \mathrm{~V}$. L'activité collective y est très réduite ( 2 minutes 43 sur 17 minutes 57 , voir aussi figure 5, lignes 2, 3 et 4), ce qui montre la part importante qu'y ont pris les élèves. Toutefois, il n'est plus question ici ni du problème de départ ni des hypothèses diverses, son enjeu réside uniquement dans les mesures, comme l'enseignant le rappelle régulièrement aux élèves (sept fois pendant les 16 minutes 25 précédant la mise en commun). Aussi n'est-il pas étonnant qu'il semble partagé par la majorité des élèves. D'ailleurs, la mise en commun après l'activité en binôme a été très brève (ligne 4) tout comme l'institutionnalisation (lignes 6 et 7), centrée sur la reprise dans le tableau préparé dans le jeu précédent des valeurs mesurées par un groupe (ligne 5), chaque binôme ayant " dû trouver sensiblement des valeurs identiques en espérant que tout le monde ait pu faire les mesures avec les deux piles " sinon il faut "prendre les valeurs de vos camarades " (min. 48:02).

Les deux jeux suivants $\mathrm{J} 7$ et J8 concernent l'exploitation des résultats, à partir de questions posées dans la fiche. Certains binômes, peu nombreux, ont pu commencer à les jouer pendant que leurs camarades terminaient leurs mesures. Probablement parce que la fin de l'heure approche, le professeur choisit de travailler uniquement de manière collective.

Si l'on se réfère à la question posée dans la fiche, le jeu 7 concerne la comparaison des tensions aux bornes des deux ampoules avec la tension aux bornes de la pile. Insatisfait de la réponse que lui livre Jérémy (voir extrait 6 , TDP 1), le professeur, en s'appuyant sur une partie de la phrase de Jérémy, poursuit un jeu différent et compare les tensions aux bornes de chaque ampoule dans un même circuit. Cette comparaison peut être rattachée à la question posée dans la séance sur la répartition des tensions, et présente donc une dissonance par rapport à ce qui figure sur la fiche élève. C'est du reste sur cette répartition que l'enseignant conclut (TDP 10). Les élèves sont partenaires a minima de ce jeu entièrement mené par l'enseignant. D'ailleurs, la formulation de la conclusion en langage naturel par les élèves est difficile si bien que l'enseignant prend là aussi une part déterminante dans l'avancée de savoirs (TDP 12 à 24).

Le jeu 8 se déroule pendant que la cloche marquant la fin du cours sonne. Son enjeu est de " trouver une relation mathématique simple (addition, soustraction...) 
Figure 5. Extrait du graphe montrant l'évolution de certains mots-clés au cours du jeu 6

(Réalisation du circuit et des mesures - min. 29:58 à 47:57)

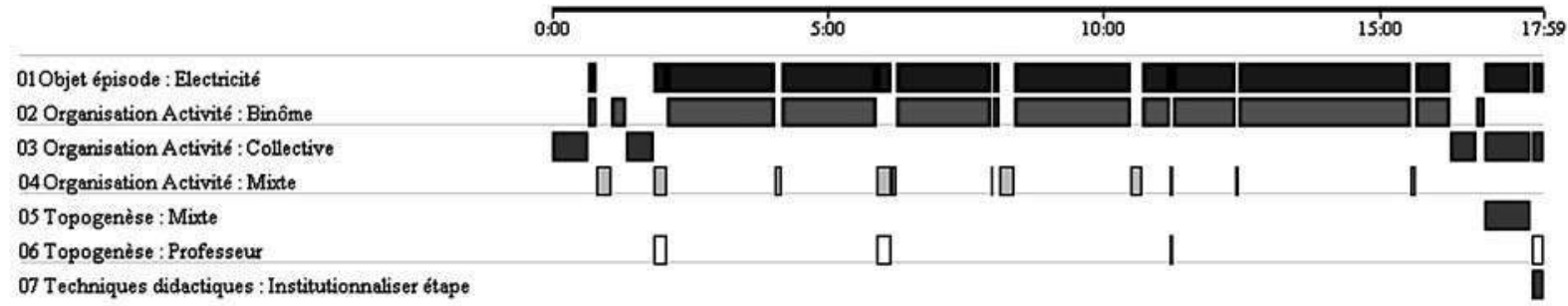

\section{Extrait 6. Mise en commun lors du jeu 7 (Comparer les tensions aux bornes de deux ampoules à celle du générateur - min. 47:58 à 51:30)}

1. Jérémy (J): L1 et L2 ne sont pas pareilles, elles n'ont pas les mêmes valeurs avec les piles de $4,5 \mathrm{~V}$ et de $9 \mathrm{~V}$.

2. $P$ : quand tu dis qu'elles sont pas pareilles, ça veut dire quoi pour toi?

3. J: ben...

4. P: est ce qu'elles sont identiques ces lampes?

5. J: non.

6. P: ce sont des lampes qui ont des tensions nominales différentes. On est tous d'accord? OK? Donc ensuite est-ce que les tensions, elles sont identiques aux bornes de ces lampes?

7. J: (Jérémy se retourne vers le tableau pour vérifier) non.

8. $\mathrm{P}$ : non d'accord. Alors la répartition des tensions est comment? Égale ou inégale ?

9. J: inégale. (les élèves sont sages mais écoutent plus ou moins)

10. $\mathrm{P}$ : on a une répartition des tensions qui se fait de manière différente en fonction...? Du dipôle considéré.

11. [....]

12. $\mathrm{P}$ : est-ce que tu pourrais faire une phrase pour dicter à tes camarades une phrase correcte en français dans le texte? Nous constatons que...

13. J: nous constatons que... L1 et L2 ne sont pas...

14. P: alors c'est L1 et L2 ou c'est les tensions?

15. J: les tensions de L1 et L2 ne sont pas inégales.

16. $\mathrm{P}$ : sont pas? Alors sont pas égales ou ne sont pas identiques.

17. $\mathrm{J}$ : ne sont pas identiques.

18. [...]

19. $\mathrm{P}$ : allez répète, demande ton camarade.

20. J: nous constatons euh nous pouvons constater nous constatons c'est pareil que les tensions L1 et L2 ne sont pas égales ou identiques.

21. P: et pourquoi elles sont pas égales, on peut expliquer pourquoi? Parce que les lampes...

22. J: parce que les lampes n'ont pas les mêmes.

23. $P$ : ne sont pas identiques.

24. J: ne sont pas identiques. 


\section{Extrait 7. Institutionnalisation de la loi d'additivité des tensions (min. 53:06 à 54:00)}

1. $\mathrm{P}:[. .$.$] et dans les deux cas qu'est-ce qu'on peut écrire? La tension aux bornes de la?$

2. E1: de la pile.

3. $\mathrm{P}$ : la tension aux bornes de la pile elle est égale à la?

4. $\mathrm{J}$ : la tension de (des élèves amènent des réponses successives inaudibles).

5. P: à la... (P montre au tableau le signe +$)$

6. Elèves: somme... (plusieurs, successivement)

7. P: la somme des?

8. Elèves: des tensions/des lampes. (en parallèle)

9. $\mathrm{P}$ : des tensions... des tensions aux bornes des lampes. On est tous d'accord ?

entre les valeurs trouvées aux bornes des dipôles et la valeur aux bornes du générateur ». Mathieu, qui est «le meilleur élève de la classe » (extrait de l'entretien), est invité à répondre en tant que « roi des mathématiques ", ce qu'il fait sans hésitation: "si on ajoute les tensions de L1 et L2, ça donne à peu près celle de la pile " (min. 52:48). La reformulation plus formalisée est ensuite opérée avec l'aide très directive de l'enseignant (voir extrait 7). Une fois que Mathieu a donné sa réponse, la topogenèse est sous la responsabilité de l'enseignant. II est difficile de dire si les élèves jouent effectivement au jeu proposé par l'enseignant ou s'ils sont dans le simple décodage des mots que ce dernier attend. Les réponses désordonnées qu'ils donnent et les formes d'interrogation de l'enseignant laissent plutôt supposer la deuxième possibilité.

\section{Un retour express sur la situation de départ}

Le jeu 9 se déroule après que la cloche a sonné une seconde fois et son enjeu est de réinvestir la loi des tensions pour comprendre la situation de départ: " Alors (en montrant l'écran sur lequel est toujours rétroprojetée la question de départ) si on prend ce circuit-là, ça règle quelque chose à votre avis? » (min. 55:00). Les élèves qui participent encore proposent d'ajouter des piles " pour avoir plus de volts ". En se situant sur le même registre que celui des élèves au début de la séance, l'enseignant suggère que l'on peut aussi « enlever des ampoules [...] pour arriver à quoi? » Pour arriver «à la tension de la pile » répondent les élèves. Cela lui permet de conclure en confondant la situation de départ et le circuit d'étude: "II faut qu'on ajoute les tensions nominales des ampoules pour que ça fasse absolument la tension de la pile, c'est ce qu'on a dit ici ( $P$ montre au tableau la loi d'additivité encadrée au tableau). La tension du générateur se répartit au niveau des différents dipôles du circuit. Ça va? » (min. 56:00).
Cette intervention clôt la leçon. Au vu des réponses dans ce jeu conclusif, il ne semble pas évident que les élèves aient davantage conscience qu'au début que la loi d'additivité des tensions soit nécessaire pour répondre au problème de départ. Celle-ci n'apparaît pas dans leur raisonnement qui semble à la fin tout aussi intuitif qu'au début. Cela nous amène à considérer, comme nous l'avons laissé entendre dans l'analyse des jeux 5 et 6 que cette séance comprend deux séries de jeux auxquels les élèves jouent de manière indépendante: la première concerne la résolution pragmatique du problème posé au départ, la seconde concerne la réalisation d'un autre circuit et des mesures associées aboutissant à la loi d'additivité.

\section{Caractéristiques essentielles de l'action didactique du professeur}

De l'analyse a priori et de l'analyse des résultats, on peut tirer un certain nombre de conclusions.

Tout d'abord, l'enseignant cherche à se conformer aux programmes. Ainsi, il cherche à mettre en place une démarche d'investigation dont on retrouve la plupart des étapes, au moins sur le plan formel, lors du déroulement de la séance. Seule la phase d'émission d'hypothèses ne trouve pas réellement sa place, même si l'enseignant suggère seulement dans ses propos qu'il l'associe à la phase de conception du circuit (extrait 4, TDP 1). Par ailleurs, l'enseignant a aussi pour objectif d'enseigner un contenu du programme, la loi d'additivité des tensions, et ce dans un temps compatible avec l'enseignement des autres contenus exigés, ce qui explique une partie de la pression temporelle sous laquelle se déroule la séance. Cette volonté existe dans la durée, puisque les modalités du fonctionnement proposé semblent intégrées au contrat didactique en vigueur. Celui-ci le conduit en particulier à proposer une situation déclenchante. 
Cette situation déclenchante est complexe et éloignée de l'enjeu de savoir. Elle est complexe parce qu'elle nécessite un raisonnement complexe pour être résolue (voir l'analyse a priori) et parce que les éléments proposés dans la solution finale sont insuffisants sur le plan scientifique pour la résoudre dans la réalité. Elle est éloignée de l'enjeu d'apprentissage parce que la loi d'additivité des tensions n'est qu'un élément parmi ceux qui interviennent dans la solution. De plus, cette situation fait l'objet d'enjeux décalés pour les élèves et l'enseignant. Pour les élèves, l'enjeu est de proposer une solution pragmatique à la situation proposée, c'est sur elle que s'opère la dévolution. En effet, on a pu voir que l'idée de rajouter des piles par exemple apparaît dans plusieurs des phases de la séance, y compris à la fin de l'enseignement. Pour l'enseignant, l'enjeu porte sur un élément de savoir à construire, la loi d'additivité des tensions, ce qui suppose de formaliser le problème. La situation entraîne l'enseignant à parler de " répartition des tensions " en lieu et place de loi d'additivité. Or la répartition des tensions (qui n'est pas un terme scientifiquement consacré) suppose l'additivité et est trop complexe pour être étudiée en $4 \mathrm{e}$.

La complexité de la situation et le décalage dans les enjeux empêchent probablement cette situation de " faire milieu » pour les élèves. Ceux-ci utilisent en effet pour s'orienter dans l'action beaucoup plus les éléments pérennes du contrat didactique ou les éléments qu'ils parviennent à décoder des attentes de l'enseignant que les relations épistémiques établies avec le milieu; la mésogenèse ainsi que la chronogenèse évoluent par à-coups et avec une discontinuité importante au jeu 4, quand le professeur passe de la raison pour laquelle la tension aux bornes des ampoules ne serait pas la bonne à la nécessité de mesurer les tensions avec un voltmètre. Pour cela, il s'appuie sur ce qui a été enseigné la séance précédente et non sur le développement du savoir en jeu à ce moment-là, d'où les discontinuités du milieu et du savoir. On retrouve également ces discontinuités dans la topogenèse. Quand le professeur laisse aux élèves la responsabilité du savoir, c'est essentiellement dans le « faire » et si les élèves sont actifs (ils répondent aux questions, ils conçoivent un circuit sur transparent, ils réalisent un circuit, ils prennent des mesures), ils élaborent peu de nouvelles idées. Certains élèves auront probablement construit une première idée de l'additivité des tensions. Peut-être aussi ont-ils construit une vision du circuit électrique en termes de tension et pas seulement en termes d'objets, mais il est impossible avec les éléments dont nous disposions de le vérifier, d'autant que si le milieu a pu rendre cette vision possible, il n'a jamais contraint les élèves à la mettre en œuvre.
II nous reste maintenant à discuter ces éléments, à la fois en s'interrogeant sur les déterminations de cette forme d'action conjointe et en revenant sur de notre question de départ.

\section{DISCUSSION}

Les déterminants que la TACD associe à l'action didactique et que l'on peut inférer de cette dernière sont déjà présents en filigrane dans la conclusion que nous venons de brosser. Ainsi l'action de l'enseignant est adressée. En effet, ce professeur cherche à être un bon sujet de l'institution " collège " (Chevallard, 2003) puisqu'il s'est engagé dans la mise en œuvre des nouveaux programmes, notamment à travers le savoir, la démarche et le type de situation qu'il propose à ses élèves ainsi que le temps consacré à cette partie. D'une autre manière, il est aussi un sujet de l'institution " enseignement de la physique » comme on peut le voir à travers le vocabulaire qu'il utilise. Dans l'enseignement de la physique en effet, jusqu'à l'introduction de la démarche d'investigation, il est question de « manipulations » et de " travaux pratiques " ce qu'on retrouve dans les propos de l'enseignant tant en classe que dans l'entretien: « en 2, [...] ce qu'on vous demande maintenant c'est la deuxième partie de votre manipulation » (par exemple, min. 14:00); « la partie 3, c'est la réalisation du TP donc tout le monde on fait comme d'habitude » (min. 28:50) ; «Pendant tout le TP, [ma préoccupation] c'était la gestion du temps " (entretien). Mais au-delà du vocabulaire, peut-être peut-on voir dans cet assujettissement une explication du fait que les élèves n'ont dans la séance que la responsabilité du " faire » (circuit, mesure), alors que l'enseignant garde la responsabilité des activités significatives sur le plan du savoir. Diverses institutions déterminent donc une partie de l'action observée en classe. Mais l'épistémologie pratique de l'enseignant a aussi un rôle important, notamment pour ce qui est du statut de l'émission d'hypothèses.

Celui-ci explique en effet dans l'entretien à propos de la proposition d'Emma qu'il n'exploite pas: « je veux pas qu'ils aient une théorie préconçue de la chose, je veux absolument qu'ils arrivent à des mesures et à partir des mesures, arriver à une conclusion; [...] je me dis, maintenant il faut que je lui fasse vérifier [à Emma], mon idée c'est de lui faire constater par l'expérience. " Les propos de l'enseignant expliquent son action didactique: le savoir scolaire se construit de manière inductive à partir de l'expérience (et peut-être aussi le savoir scientifique). Or, la démarche d'investigation 
encourage les élèves à formuler "des hypothèses explicatives » que l'on peut considérer, même si ce n'est pas réellement explicité dans les textes, comme des modèles hypothétiques permettant de faire des prévisions qu'il s'agit ensuite de tester par un dispositif expérimental adéquat. Ce sont donc bien des « théories préconçues " que les élèves devraient formuler en suivant cette démarche, qui pour l'enseignant n'ont pas leur place. Ce positionnement épistémologique observé dans la pratique, hétérodoxe par rapport aux analyses de l'épistémologie contemporaine, ne semble pas particulier à cet enseignant. Ainsi, en ce qui concerne les hypothèses, Mathé relève que les enseignants concernés par son étude doctorale désignent par ce terme aussi bien une proposition de dispositif expérimental permettant d'obtenir un effet souhaité qu'une prévision non fondée sur un modèle explicatif (2010, p. 49, p. 150, p. 175). Gyllenpalm, Wickman et Holmgren (2010) relèvent une situation semblable en Suède: " the teachers seemed to use "hypothesis" as meaning an educated guess about what might happen in a laboratory task or exercise [...] The function given to this term by the teachers seemed to be synonymous with that of a "prediction". " Par ailleurs, de manière plus générale, selon de nombreuses études basées sur des méthodologies déclaratives (par exemple Lederman, 1992; Abd-El-Khalick \& Lederman, 2000; Porlan-Ariza, Garcia, Rivero Garcia et al., 1998; Robardet, 1998; Roletto, 1998), les points de vue épistémologiques des enseignants sont hétérogènes, constitués d'éléments empruntés à différents modèles épistémologiques. Ils relèveraient généralement de l'empirisme quant au statut des connaissances auxquelles ils confèrent une supériorité par rapport aux connaissances non scientifiques, au réalisme quant à leur rapport aux objets dont elles parlent et à l'inductivisme pour ce qui est de leur processus d'élaboration (Pélissier $\&$ Venturini, 2012). De plus, ils minimiseraient la valeur des connaissances scientifiques comme instruments de prévision et d'explication. Tout cela n'est pas sans faire écho aux conclusions précédentes.

De plus, au vu de l'analyse que nous avons faite de la situation proposée et des savoirs qu'elle met en jeu, nous considérons de manière plus large que le rapport personnel (Chevallard, 2003) que l'enseignant entretient non seulement avec la démarche développée mais aussi avec le savoir et la situation en jeu ne le conduit à mesurer ni l'ensemble des liens existants entre ces trois éléments ni leur nature. II est vrai que l'institution ne favorise pas les choses puisque la cohérence à introduire entre savoir, démarche et situation est laissée à la charge de l'enseignant. Ainsi par exemple, si l'enseignant utilise une situation "déclenchante ", il doit s'assurer que celle-ci est bien cohérente avec le savoir des programmes, ce qui n'est pas le cas ici, puisque la situation amène l'enseignant à évoquer la "répartition des tensions " alors que le savoir visé est simplement leur additivité. Ainsi, ces rapports personnels seraient aussi selon nous des déterminants de l'action didactique.

Les différents aspects que nous venons d'évoquer ne sont pas sans faire écho aux conclusions proposées par Calmettes (2009) à la suite d'une des rares études qui, à notre connaissance, ait examiné les pratiques d'enseignants français ordinaires en sciences physiques au collège. Selon ses conclusions en effet, " la construction du milieu didactique constitue [...] un obstacle à la mise en œuvre de la démarche d'investigation ", certains enseignants " proposant un problème pour lequel il n'y pas de cohérence entre consigne, prérequis, gestion du temps et dispositif d'aide ", "la référence de l'activité proposée étant inductiviste tel que le définit Chalmers (1987, p. 20), "la science commençant par l'observation" ", avec devant les difficultés, "un retour à la coutume didactique dans laquelle l'enseignant utilise un guidage strict des activités ».

Enfin, pour terminer, nous constatons que le rapport personnel que les élèves entretiennent avec la situation en jeu (et peut-être de manière plus générale avec les aspects empiriques) pèse sur l'action didactique. Ils proposent en effet à plusieurs reprises d'enlever des ampoules et de rajouter une batterie (ou de la remplacer par une batterie plus "forte") pour obtenir un éclairage convenable dans la cabane. Il s'agit là d'une solution pragmatique, immédiate, très liée au problème posé, relevant du sens commun et des modes de fonctionnement de la vie quotidienne. Ce comportement est compatible avec le savoir en jeu dans la classe au début de la séance (min. 6:54) lorsqu'il s'agit pour eux de s'approprier la situation en vue de formuler une question scientifique à résoudre; il l'est moins au milieu de la séance (min. 27:15) lorsque la classe a entamé le travail d'élaboration d'un modèle susceptible d'être valide dans une classe de situations; il l'est encore moins à la fin de la séance (min. 55:32) lorsque l'enseignant revient sur la situation de départ avec l'objectif d'utiliser le modèle établi (la loi des tensions) pour interpréter cette situation et décider de l'action à tenir, en leur demandant ainsi (mais implicitement) d'adopter une posture scientifique qu'ils ne prendront pas. C'est en ce sens que nous défendons l'idée que le rapport personnel des élèves à la situation proposée, très liée à la vie quotidienne, détermine en bonne partie leur action, même si par ailleurs, ils ont très consciencieusement réalisé le circuit et les mesures demandés. Bien sûr l'adoption explicite de la posture scientifique ne peut résulter que d'une construction 
progressive impliquant l'aide de l'enseignant, cette construction n'est pas en jeu dans la chronogenèse de cette séance. Cela renvoie donc probablement aussi à l'épistémologie pratique de ce dernier, notamment à sa conception de la nature des savoirs scientifiques.

Si cette première partie de la discussion permet de comprendre en partie les observations réalisées au cours de cette séance à travers les déterminants que nous venons de pointer, elle permet aussi de revenir à travers cette étude de cas, sur la question de départ, plus générale: qu'en est-il après trois ou quatre ans de la mise en œuvre des nouveaux programmes d'enseignement des sciences physiques et chimiques du collège? Tout d'abord, il est évident que cet enseignant tente de mettre en œuvre ces nouveaux programmes et qu'il accepte de prendre les risques correspondants pour se conformer à la réforme exigée. II n'est d'ailleurs pas du tout seul à adopter cette posture comme en témoignent les très nombreux contacts professionnels que nous avons eus avec les enseignants de la discipline. En cela, on peut faire l'hypothèse que des enseignants contredisent la conclusion de Gather Thurler selon laquelle les réformes effectuées par le biais de textes ministériels, liées à la redéfinition des rôles et des fonctions des acteurs du système éducatif ne parviennent pas à développer " la volonté d'apprendre et le goût du risque qui sont indispensables pour changer » (2000, p. 29). On peut toutefois penser que la situation critique dans laquelle se trouve l'enseignement scientifique actuel, pointée par de nombreux rapports - par exemple, de l'OCDE (2008) ou, en France, d'Ourisson (2002) - et dont les enseignants ont aussi conscience, est un moteur puissant pour oser l'innovation proposée, au-delà de la volonté de satisfaire l'institution. En même temps, conformément à l'analyse de Crahay, Wanlin, Issaieva et alii (2010, p. 111), on voit comment certaines croyances ou connaissances de cet enseignant, explicitées ici en termes de rapports personnels ou d'épistémologie pratique constituent un filtre à l'égard des exigences et de la philosophie des nouveaux programmes, voire sont en tension ou en opposition avec elles, et en limitent donc pour l'instant considérablement la portée. Si ces connaissances ou croyances déterminent les pratiques, elles sont aussi en grande partie construites à partir de la pratique (Sensevy, 2007 , p. 37) et sont donc liées pour une bonne part à l'institution " enseignement des sciences physiques et chimiques". Aussi, nous faisons l'hypothèse que le cas de cet enseignant n'est pas isolé, qu'il est probablement similaire au cas d'autres enseignants de la discipline à la suite de l'évolution des programmes instituant à chaque fois que c'est possible l'usage de la démarche d'investigation dans l'enseignement primaire et secondaire.
II reste alors à s'interroger, même si ce n'est pas l'objet de cet article, sur la manière de faire évoluer progressivement et de concert, connaissances/ croyances et pratiques. L'expérience que nous avons (Tiberghien, Vince \& Gaidoz, 2009; Tiberghien, 2011) mais aussi d'autres travaux (Brand \& Moore, 2011; Howe \& Stubbs, 1997) laissent supposer que l'existence d'un collectif d'enseignants travaillant sur la conception d'un ensemble de séances et l'analyse de leur mise en œuvre, inscrit dans la durée et accompagné par un chercheur ou un formateur s'appuyant sur les acquis de la recherche est une solution possible. À travers cette étude, on peut faire l'hypothèse que les questions liées à la nature des savoirs scientifiques, la question des références potentielles (psychologiques et épistémologiques) de cette démarche, la question de la problématisation, par exemple, pourraient être des points d'ancrage significatifs pour travailler à la conception, la mise en œuvre et l'analyse de séances dans le travail de ces collectifs.

\section{CONCLUSION}

À la suite de la publication de nouveaux programmes instituant des changements importants dans la manière d'enseigner les sciences physiques et chimiques, nous avons souhaité examiner leur mise en œuvre. Pour cela nous avons analysé la pratique d'un enseignant de terrain à l'aide de la TACD. Cette étude de cas a concerné une séance d'enseignement de la loi d'additivité des tensions dans une classe de $4^{\mathrm{e}}$ à l'aide de la démarche d'investigation par un jeune enseignant en début de carrière. Elle a permis de montrer à la fois sa volonté de satisfaire aux exigences institutionnelles et la difficulté à le faire: situation déclenchante complexe et non directement liée aux savoirs en jeu, décalage entre les enjeux de cette situation pour les élèves et pour l'enseignant, responsabilité des activités les plus significatives sur le plan épistémique assurée par l'enseignant, les élèves ayant des responsabilités dans les tâches plus secondaires, action des élèves souvent orientée à partir des éléments pérennes du contrat didactique et non par les relations épistémiques établies avec le milieu, statut particulier donné à l'émission d'hypothèses. De ces observations et de l'entretien de type autoconfrontation avec l'enseignant, nous avons inféré des déterminants de l'action didactique: action adressée à différentes institutions, épistémologie pratique, rapports personnels aux savoirs concernés, à la situation proposée et à la démarche mise en œuvre. En considérant qu'une bonne part de ces éléments est liée 
à l'institution « enseignement des sciences physiques et chimiques » mais aussi à partir des nombreux contacts que nous avons avec le terrain, nous faisons l'hypothèse que les conclusions de cette étude de cas ont une portée plus générale quant aux difficultés vécues par les enseignants pour rénover l'enseignement scientifique selon les prescriptions qui leur sont faites actuellement. Cela nous amène à conclure qu'une rénovation de fond comme celle qui est envisagée, sûrement nécessaire, pourrait être envisagée avec profit dans le cadre de collectifs d'enseignants, accompagnés dans la durée, et beaucoup moins dans le cadre de formations individuelles, courtes et ponctuelles comme c'est souvent le cas à l'heure actuelle dans certaines académies.
La recherche en éducation scientifique commence à identifier les difficultés des enseignants face à la mise en œuvre de ces nouveaux programmes; elle a dans la mise en place des formations correspondantes et leur évaluation un nouveau champ d'investigation sûrement aussi important.

Patrice Venturini patrice.venturini@univ-tlse2.fr Université de Toulouse-Le Mirail

Andrée Tiberghien andree.tiberghien@univ-lyon2.fr CNRS-Université de Lyon

\section{NOTES}

1 HUBERMAN A. M. (1973). "Comment s'opèrent les changements en éducation : contribution à l'étude de l'innovation ". Expérience et innovation en éducation, $\mathrm{n}^{\circ} 4$.

2 WITTGENSTEIN L. ([1953] 1997). Philosophical Investigations. Oxford: Blackwell.

3 DOUGLAS M. (1987). How institutions think. Londres : Routledge.

4 DOUGLAS M. (1996). Thought styles. Londres: Sage Publications.

5 FLECK L. ([1934] 1979). Genesis and development of a scientific fact. Chicago : The University of Chicago Press.
6 BOURDIEU P. (1992). Language and Symbolic Power. Cambridge : Polity Press.

7 La définition de Brousseau inclut seulement l'élève. Nous y ajoutons l'enseignant, en référence à l'action conjointe.

8 Voir en ligne : http://visa.inrp.fr

9 Voir en ligne : http://www.transana.org/

10 L'indication suivante « 3,5 V 0,2 A » proposée sur le culot de l'ampoule indique sa tension et son intensité nominales.

\section{BIBLIOGRAPHIE}

ABD-EL-KHALICK F. \& LEDERMAN N. (2000). « Improving science teachers' conceptions of nature of science: A critical review of the litterature ". International Journal of Science Education, no 22-7, p. 665-701.

ALLEVARD M., CARRON R., MARTIN D., COLONNA A.M., MARQUIS E., TOURNIER G., CHAMPAGNON B. \& TRIBOLLET B. (1991). «Les erreurs en électrocinétique : identification et analyse ». Bulletin de l'union des physiciens, no 730 , p. $145-158$.

AMADE-ESCOT C. \& VENTURINI P. (2009). « Le milieu didactique : d'une étude empirique en contexte difficile à une réflexion sur le concept ". Éducation \& didactique, vol. 3, no 1, p. 7-43.

BLANCHARD M. R., SOUTHERLAND S. A., OSBORNE J. W., SAMPSON V. D., ANNETTA L. A. \& GRANGER E. M. (2010). "Is inquiry possible in light of accountability?: A quantitative comparison of the relative effectiveness of guided inquiry and verification laboratory instruction ». Science Education, vol. 94, no 4, p. 577-616.

BRAND B. R. \& MOORE S. J. (2011). «Enhancing teachers' application of inquiry-based strategies using a constructivist sociocultural professional development model ». International Journal of Science Education, vol. 33, no 7, p. 889-913.

BROUSSEAU G. (2003). Glossaire de quelques concepts de la théorie des situations didactiques en mathématiques. En ligne : http://pagesperso-orange.fr/daest/guy-brousseau/ textes/Glossaire_Brousseau.pdf (consulté le 1er août 2011).

BENSEGHIR A. (1988). " Formation des concepts d'électrocinétique : un point de vue historique ". Technologies, idéologies, pratiques, vol. 7, n० 2, p. 7-21.

CALMETTES B. (2009). " Démarches d'investigation en physique. Des textes officiels aux pratiques en classe ". Spirale, no 43, p. 139-148.

CHALMERS A. (1987). Qu'est-ce que la science ? Paris : La Découverte.

CHEVALLARD Y. (2003). " Approche anthropologique du rapport au savoir et didactique des mathématiques ». In S. Maury \& M. Caillot (dir.), Rapport au savoir et didactiques. Paris : Fabert, p. 81-122.

CHINN C. A. \& MALHOTRA B. A. (2002). « Epistemologically authentic inquiry in schools: A theoretical framework for evaluating inquiry tasks ". Science Education, vol. 86, n 2, p. $175-218$.

CLOSSET J.-L. (1989). « Les obstacles à l'apprentissage de l'électrocinétique ». Bulletin de l'union des physiciens, no 716, p. 931-949.

CRAHAY M., WANLIN P., ISSAIEVA É. \& LADURON I. (2010). "Fonction, structuration et évolution des croyances (et connaissances) des enseignants ". Revue française de pédagogie, no 172, p. 85-129. 
CROS F. (1997). « L'innovation en éducation et en formation ». Revue française de pédagogie, no 118, p. 127-156.

DELL'ANGELO M., COQUIDÉ M. \& MAGNERON N. (2012). " Statut de l'investigation dans des standards de l'enseignement scientifique : cas des USA, de la Suisse et de la France ". In B. Calmettes (dir.), Didactique des sciences et démarches d'investigation. Références, représentations, pratiques et formation. Paris : L'Harmattan.

FURTAK E. M. (2006). " The problem with answers: An exploration of guided scientific inquiry teaching ". Science Education, no 90, p. 453-467.

GATHER THURLER M. (2000). “L'innovation négociée : une porte étroite ». Revue française de pédagogie, no 130, p. $29-42$.

GESS-NEWSOME J. (2002). « The use and impact of explicit instruction about the nature of science and science inquiry in an elementary science methods course ». Science and Education, vol. 11, p. 55-67.

GYLLEMPALM J., WICKMAN P. O \& HOLMGREN S. O. (2010). " Teachers' language on scientific inquiry: Methods of teaching or methods of inquiry? ». International Journal of Science Education vol. 32, no 9, p. 1151-1172.

GRANDY R. \& DUSCHL R. (2007). " Reconsidering the Character and Role of Inquiry in School Science: Analysis of a Conference ". Science \& Education, vol. 16, n० 2, p. 141-166.

HOWE A. C. \& STUBBS H. S. (1997). « Empowering science teachers: A model for professional development ". Journal of Science Teacher Education, vol. 8, n 3, p. 167-182.

KRAJCIK J. S., BLUMENFELD P. C., MARX, R. W., BASS K. M., FREDERICKS J. \& SOLOWAY E. (1998). « Inquiry in projectbased science classrooms: Initial attempts by middle school students ». Journal of the Learning Sciences, vol. 7 , n० 34, p. 313-350.

JOHSUA S. (1989). " Le rapport à l'expérimental dans la physique de l'enseignement secondaire ". Aster, n० 8, p. 30-53.

LEBLANC S., RIA L. \& VEYRUNES P. (à paraître). « Vidéo et analyse in situ des situations d'enseignement et de formation dans le programme du cours d'action ». In L. Veillard \& A. Tiberghien (dir.), Instrumentation de la recherche en Éducation. Le cas du développement d'une base de vidéos de situation d'enseignement et d'apprentissage ViSA. Paris : Maison des sciences de l'homme.

LEDERMAN, N. G. (1992). « Students' and teachers' conceptions of the nature of science: a review of the research ». Journal of Research of Science Teaching, vol. 29, no 4, p. 331-359.

LIGOZAT F. \& SCHUBAUER-LEONI M.-L. (2009). « The joint action theory in didactics: Why do we need it in the case of teaching and learning mathematics? ». In Proceeding of the Sixth Conference of European Research in Mathematics Education (January 28th-February 1st 2009). Lyon : INRP, p. 83-92. En ligne : http://www.inrp.fr/publications/editionelectronique/cerme6/wg9-09-ligozat.pdf (consulté le $1^{\text {er }}$ août 2011).

LINN M. C., DAVIS E. A. \& BELL P. (2004). Internet environments for science education. Mahwah, NJ : Erlbaum.

MAKITALO-SIEGL K., KOHNLE C. \& FISCHER F. (2011). " Computer-Supported Collaborative Inquiry Learning and Classroom Scripts: Effects on Help-Seeking Processes and Learning Outcomes ». Learning and Instruction, vol. 21, no 2 , p. 257-266.
MATHÉ S. (2010). La démarche d'investigation dans les collèges français : élaboration d'un dispositif de formation et étude de l'appropriation de cette nouvelle méthode d'enseignement pas les enseignants. Thèse de doctorat, didactique des disciplines, université de Paris 7.

MATHÉ S., MÉHEUT M. \& De HOSSON C. (2008). « Démarche d'investigation au collège : quels enjeux ? ». Didaskalia, no 32 , p. 41-76.

MINISTÈRE DE L'ÉDUCATION NATIONALE (2007). "Programmes de l'enseignement des mathématiques, des SVT, de physique-chimie du collège ». Bulletin officiel spécial, 19 avril 2007, n 6.

MINISTÈRE DE L'ÉDUCATION NATIONALE (2008). « Programme du collège ». Bulletin officiel spécial, 28 août 2008, n 6 .

MONOD-ANSALDIR. \& PRIEURM. (coord.) (2011). « Démarches d'investigation dans l'enseignement secondaire représentations des enseignants de mathématiques, SPC, SVT et technologie ». Rapport d'enquête Ifé-ENS Lyon. Lyon : Ifé-ENS de Lyon.

ORGANISATION DE COOPÉRATION ET DE DÉVELOPPEMENT ÉCONOMIQUE (2008). Encouraging student interest in science and technology studies: global science forum. Paris : OCDE.

OURISSON G. (2002). Désaffection des étudiants pour les études scientifiques. Paris : Ministère de l'Éducation nationale.

PÉLISSIER L. \& VENTURINI P. (2012). « Qu'attendre de la démarche d'investigation en matière de transmission savoirs épistémologiques ? ". In B. Calmettes (dir.), Didactique des sciences et démarches d'investigation. Références, représentations, pratiques et formation. Paris : L'Harmattan.

PORLAN ARIZA R., GARCIA E., RIVERO GARCIA A. \& MARTIN del POZO R. (1998). " Les obstacles à la formation professionnelle des professeurs en rapport avec leurs idées sur la science, l'enseignement et l'apprentissage ». Aster, no 26, p. 207-235.

REID N. \& YANG M. J. (2002). « Open-ended problem solving in school chemistry: A preliminary investigation ». International Journal of Science Education, vol. 24, n², p. 1313-1332.

ROBARDET G. (1998). " La didactique dans la formation des professeurs de sciences physiques face aux représentations sur l'enseignement scientifique ». Aster, no 26, p. 31-58.

ROEHRIG G. H. \& LUFT J. L. (2004). « Constraints experienced by beginning secondary science teachers in implementing scientific inquiry lessons ». International Journal of Science Education, vol. 26, n० 1, p. 3-24.

ROLETTO E. (1998). " La science et les connaissances scientifiques ». Aster, n०26, p. 11-30.

SAMPSON V., GROOMS J. \& WALKER J. P. (2011). «ArgumentDriven Inquiry as a way to help students learn how to participate in scientific argumentation and craft written arguments: An exploratory study ». Science Education, vol. 95, no 2, p. 217-257.

SANDOVAL W. A. (2005). "Understanding students' practical epistemologies and their influence on learning through inquiry ». Science Education, vol. 89, n० 4, p. 634-656.

SCHWARTZ R. S., LEDERMAN N. G \& CRAWFORD B. A. (2004). « Developing views of nature of science in an authentic context: An explicit approach to bridging the gap 
between nature of science and scientific inquiry ». Science Education, vol. 88, no 4, p. 610-645.

SENSEVY G. (2007). «Des catégories pour décrire et comprendre l'action didactique ». In G. Sensevy \& A. Mercier (dir.), Agir ensemble : l'action didactique conjointe du professeur et des élèves. Rennes : Presses universitaires de Rennes, p. 13-49.

SENSEVY G. (2009). « Outline of a joint action theory in didactics ». In Proceedings of the Sixth Conference of European Research in Mathematics Education (January $28^{\text {th }}$ February 1st 2009). Lyon : INRP, p. 113-122. En ligne : http:// www.inrp.fr/publications/edition-electronique/cerme6/ wg9-12-sensevy.pdf (consulté le 1er août 2011).

SENSEVY G. (2011). Le sens du savoir. Éléments pour une théorie de l'action conjointe en didactique. Bruxelles : De Boeck.

SHIMODA T. A., WHITE B. Y. \& FREDERIKSEN J. R. (2002). "Student goal orientation in learning inquiry skills with modifiable software advisors ». Science Education, vol. 86, n०2, p. 244-263.

SMITHENRY D. W. (2010). «Integrating Guided Inquiry into a Traditional Chemistry Curricular Framework ». International Journal of Science Education, vol. 32, no 13, p. 1689-1714.

TIBERGHIEN A., VINCE J. \& GAIDOZ P. (2009). « Design based research. Case of teaching sequence on mechanics ". International Journal of Science Education, vol. 31, no 17, p. 2275-2314.

TIBERGHIEN A., MALKOUN L. \& SECK M. (2008). «Analyse des pratiques de classes de physique : aspects théoriques et méthodologiques ". Les Dossiers des sciences de l'éducation, no 19, p. 61-79.

TIBERGHIEN A. (2011). "Conception et analyse de ressources d'enseignement : le cas des démarches d'investigation ». In M. Grangeat (dir.), Les démarches d'investigation dans l'enseignement scientifique. Pratiques de classe, travail collectif enseignant, acquisitions des élèves. Lyon : INRP.
VEILLARD L. \& TIBERGHIEN A. (à paraître). Instrumentation de la recherche en Éducation. Le cas du développement d'une base de vidéos de situation d'enseignement et d'apprentissage ViSA. Paris : Maison des sciences de I'homme.

VENTURINI P. (org.) (2010). « Analyse croisée d'un même corpus vidéo et des documents associés appartenant à une base de vidéos de situations d'enseignement apprentissage (ViSA) : conditions de possibilité, limites, objets-frontière ". In Résumé des communications du congrès Actualité de la recherche en éducation et formation, organisé à Genève du 13 au 16 septembre 2010. Genève : Université de Genève, p. 299-301.

VENTURINI P. \& TIBERGHIEN A. (2012). «Potential Learning Outcomes Inferred from French Curricula in Science Education ». In S. Bernholt, K. Neumann, \& P. Nentwig (dir.), Making It Tangible - Learning Outcomes in Science Education. Münster: Waxmann, p. 475-509.

WALKER K. A. \& ZEIDLER D. L. (2007). "Promoting Discourse about Socioscientific Issues through Scaffolded Inquiry ». International Journal of Science Education, vol. 29, no 11, p. $1387-1410$.

WATSON R. J., SWAIN J. R. L. \& Mc ROBBIE C. (2004). "Students' discussions in practical scientific inquiries". International Journal of Science Education, vol. 26, no 1 , p. $25-45$.

WU H. \& KRAJCIK J. S. (2006). « Inscriptional Practices in Two Inquiry-Based Classrooms: A Case Study of Seventh Graders' Use of Data Tables and Graphs ». Journal of Research in Science Teaching, vol. 43, no 1, p. 63-96.

YAGER R. (1997). « Science education a science? ». Electronic Journal of Science Education, vol. 2, no 1-4. En ligne : http://wolfweb.unr.edu/homepage/jcannon/ejse/yager.html (consulté le 2 avril 2012).

Van ZEE E. (2000). "Analysis of a student-generated inquiry discussion ». International Journal of Science Education, vol. 22, no 2, p. 115-142. 


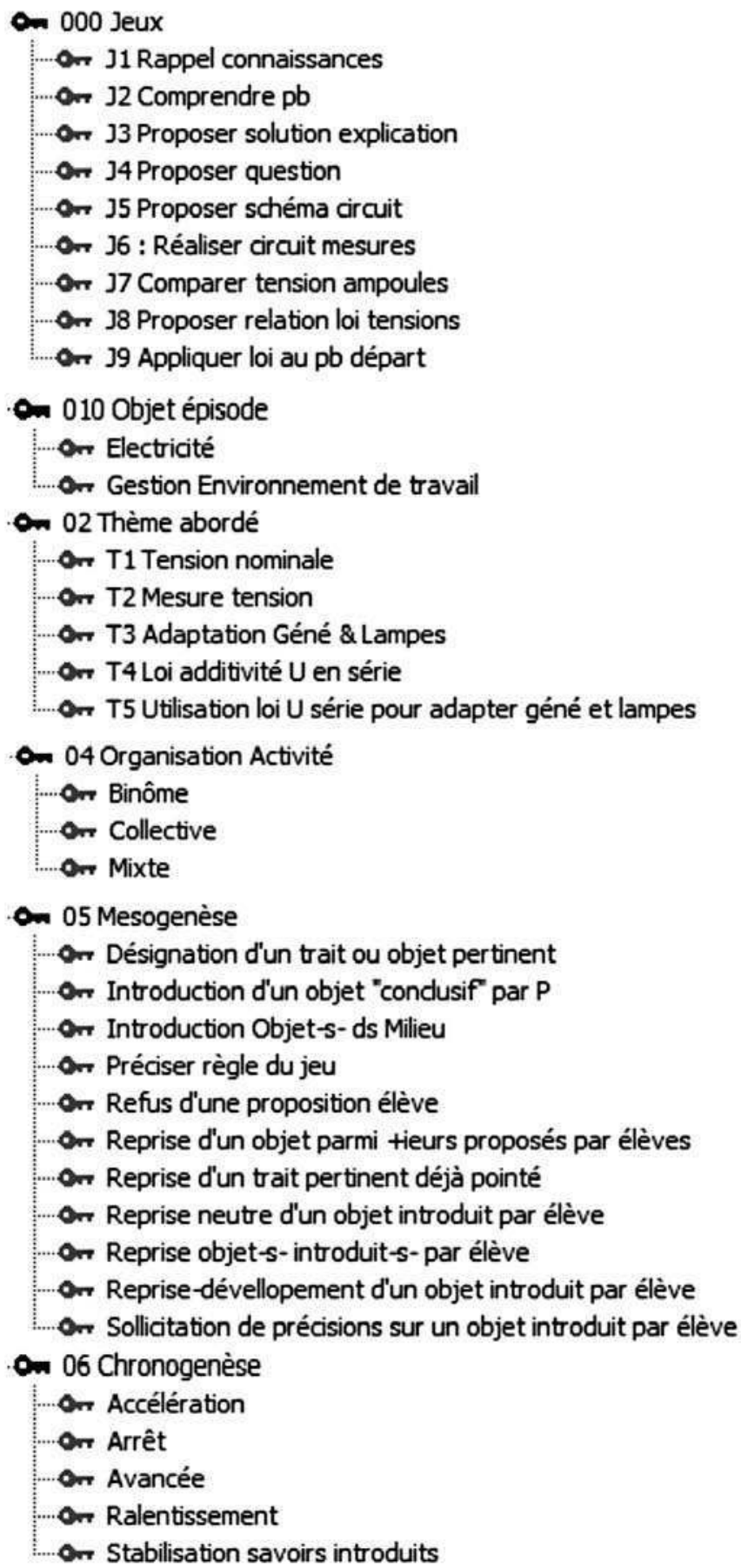




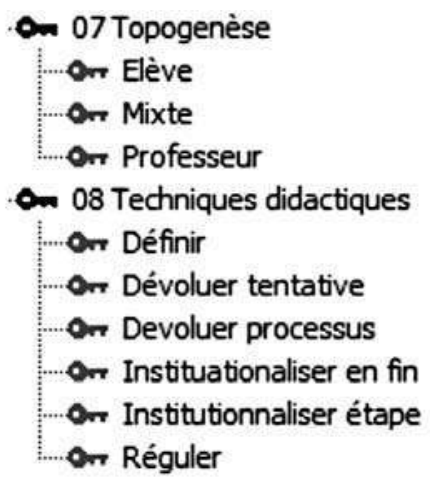

